*For correspondence: lindmuir@umich.edu (LAM)

\title{
Obesity disrupts innate-adaptive immune network patterning in adipose tissue
}

4 Gabrielle A. Dotson ${ }^{1}$, Indika Rajapakse ${ }^{1,2}$, Lindsey A. Muir ${ }^{1 *}$

5 'Department of Computational Medicine and Bioinformatics, University of Michigan, - Ann Arbor, Michigan; ${ }^{2}$ Department of Mathematics, University of Michigan, Ann Arbor, Michigan

9

\begin{abstract}
Obesity drives significant changes in adipose tissue that precede development of tissue and systemic insulin resistance. Immune cell infiltration and inflammation are known contributors to these changes but there is limited understanding of their spatial context tissue-wide. We sought to identify the spatial patterning of adipose tissue immune cells in obesity. We collected epididymal adipose tissue from mice in a time course of diet-induced obesity, integrating spatial transcriptomics and single-cell RNA-sequencing to identify dominant cell type signatures preserved in their anatomical context. Our integrated analyses enable deconvolution of cell types at spatial data spots, quantitation of gene expression patterns at spots throughout adipose tissue, cell type network analysis, and investigation of ligand-receptor colocalization. Our data support increased innate immune cell quantity with tissue-wide interspersion and dampened adaptive immune cell signatures with obesity. Furthermore, network analyses identify increased heterogeneity within all major immune cell types, suggesting patterning consistent with increased numbers of subtypes. By describing the position-specific cellular composition of adipose tissue in mice for the first time, we provide a framework for better understanding cell cooperation toward emergence of multicellular tissue function.
\end{abstract}

\section{Introduction}

Global increases in obesity raise significant concern about the development of cardiovascular and metabolic disease, increased risk of adverse events in viral infection, and economic and health care costs (Misumi et al., 2019; Dai et al., 2020). Striking changes in white adipose tissue (WAT) size and cellular content are observed in obesity, including shifts in immune cell types and polarization that are associated with metabolic dysfunction in mice and humans (Lumeng et al., 2007, 2008; Muir et alo, 2018, 2022). Dramatic changes in the quantity of some stromal vascular cell (SVC) populations occur in the first few weeks of high-fat diet (HFD)-feeding in mice, aligning with changes in adipocyte hypertrophy (Muir et al., 2018). Despite population and single-cell transcriptomics that enable low-bias characterization of these obesity-related changes in WAT immune cells Vaitin et al., 2019; Vijay et al., 2020; Muir et al., 2022), the molecular events triggering tissue dysfunction remain poorly understood and include important anatomical contexts that are lost with tissue digestion. Tissue immunostaining methods capture anatomical context but with a practical bias in selection of a small number of markers that can be used concurrently, which is especially problematic for distinguishing cells that express similar surface proteins such as in the myeloid lineage. Spatial transcriptomics now enables mapping of gene expression across tissue sections, preserv- 
ing spatial relationships while reducing artifacts introduced by tissue digestion and downstream cell processing.

In the case of immune cells in adipose tissue, using one or two markers is insufficient to identify subtypes that could have functional distinctions relevant to disease etiology, progression, and prognosis. Myeloid cells in adipose tissue, such as macrophages (ATMs) and dendritic cells (ATDCs), have roles in tissue homeostasis and contribute to development of insulin resistance in murine obesity. Complex gating schemes with many markers are used in flow cytometry to reliably identify ATM and ATDC subtypes, but ICC cannot accommodate the same. Spatial transcriptomics has the potential to provide reliable identification of these populations in anatomical context at $55 \mu \mathrm{m}$ resolution.

The aim of the present study is to spatially map the microenvironment of mouse adipose tissue for the first time. We present the tissue landscape as a network of cells and interrogate how spatial patterning and cell-cell communication, especially as it relates to immune response, trend with progressing obesity in diet-controlled mice. Prior studies incorporating diet-induced obesity models and transcriptional profiling of adipose tissue have provided valuable insight into hallmarks of obesity like the development of insulin resistance (Uysal et al., 1997), macrophage accumulation (Weisberg et al., 2003; Lumeng et al., 2007), and increasing cellular diversity. However, due to experimental techniques that rely on tissue dissociation and homogenization, these studies fail to demonstrate the contribution of spatial organization to adipose tissue dynamics. More recently, spatial transcriptomics (Ståhl et al., 2016) was used to study adipocyte subpopulations in human adipose tissue (Bäckdahl et al., 2021), revealing differential responses to insulin stimulation among three distinct subtypes. The full breadth of immune cell populations in adipose tissue, however, remains to be explored in a spatial context. Moreover, our present study provides a temporal axis to study how adipose tissue landscape changes with obesity.

\section{Results}

\section{Lean and obese adipose tissue landscape}

We evaluated tissue and metabolic function in our diet-induced obesity model using mice fed a normal diet (ND), a high-fat diet for 8 weeks ( $8 w$ ), and a high-fat diet for 14 weeks (14w) (Figure 1A). As expected, mice fed a HFD gained body and epididymal white adipose tissue (eWAT) weight (Figure 1B-D). Glucose tolerance tests (GTT) showed increased area under the curve (AUC) starting at one week of HFD feeding, with the highest AUC and variability at intermediate time points (Figure 1E,F). Adipocyte sizing showed increased frequency of large adipocytes and greater mean adipocyte size in the $8 \mathrm{w}$ and $14 \mathrm{w}$ cohorts (Figure $1 \mathrm{G}, \mathrm{H}$ ). These data are consistent with pre-diabetes of this HFDfed model, showing early insulin resistance during HFD feeding and a period of variable insulin resistance that aligns with dynamic restructuring of adipose tissue.

Changes in SVCs in murine diet-induced obesity are well documented and include immune cell infiltration, proliferation, and altered immune cell cross-talk that disrupt homeostatic mechanisms (Morris et al., 2013; Muir et al., 2018; Porsche et al., 2020, 2021). In this model, the largest changes in cellularity occur during early adipose tissue expansion and keep pace with changes in adipocyte size (Muir et al., 2018). We sought to capture this changing landscape and altered cell-cell communication by spatiotemporal molecular profiling, which can uncover patterns as tissue function breaks down (Asp et al., 2019; Chen et al., 2020). Here we profiled genome-wide transcripts across eWAT sections from ND and HFD-fed mice using spatial transcriptomics (Ståhl et al., 2016; Bäckdahl et alo, 2021) via the Visium Spatial Gene Expression platform. Examining these data, we found increased Ptprc (CD45), which is broadly expressed in immune cells, and increased ATM-associated CD68. Notably, we observed increased pro-inflammatory ATM marker Itgax (CD11C), resident ATMassociated marker Mrc1, and Trem2, which is expressed in lipid-associated ATMs and has proposed functions in protective lipid homeostasis and adipose tissue remodeling in obesity (Jaitin et al., 2019; Liu et al., 2019; Sharif et al., 2021) (Figure 11,J). 
bioRxiv preprint doi: https://doi.org/10.1101/2022.02.26.482134; this version posted February 27, 2022. The copyright holder for this preprint (which was not certified by peer review) is the author/funder. All rights reserved. No reuse allowed without permission.

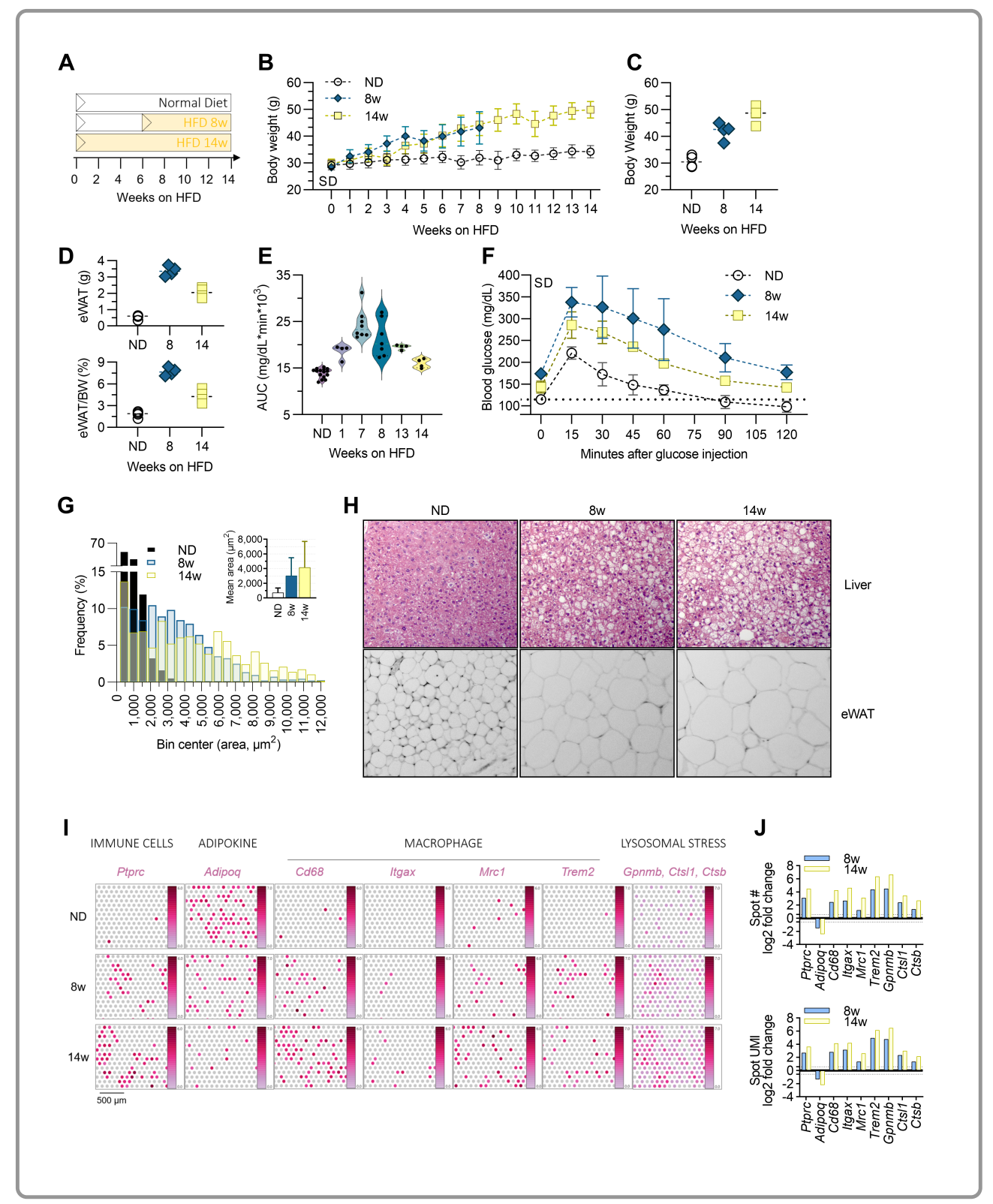

Figure 1. Diet-induced obesity and adipose tissue remodeling. (A) Time course for mice fed a 60\% high-fat diet (HFD) for 8 weeks (8w) or 14 weeks (14w), vs. normal diet (ND) controls. Measurements included weight gain (B), final body weight (C), epididymal adipose tissue (eWAT) weight and eWAT as a percentage of body weight (D), and glucose tolerance test data showing area under the curve (AUC) across time points (E) and glucose measurements for cohorts one week prior to endpoint tissue collection (F). (G) Frequency distribution and average adipocyte size in eWAT of ND, 8w, and $14 \mathrm{w}$ cohorts. (H) Representative images from H\&E staining of eWAT and liver. (I) Representative maps showing spatially preserved gene expression in eWAT. Each spot is $55 \mu \mathrm{m}$ diameter location of transcript capture from a tissue section, colored corresponding to the expression level ( $\log _{2}$ transformed) of select obesity-related genes. $(\mathbf{J})$ Gene expression changes in HFD-fed mice compared to ND mice corresponding to the genes shown in part (I). Upper graph, $\log _{2}$ fold change in the quantity of spots in HFD cohorts; Lower graph, $\log _{2}$ fold change in average tissue-wide expression of a gene in HFD cohorts. 
bioRxiv preprint doi: https://doi.org/10.1101/2022.02.26.482134; this version posted February 27, 2022. The copyright holder for this preprint (which was not certified by peer review) is the author/funder. All rights reserved. No reuse allowed without permission.

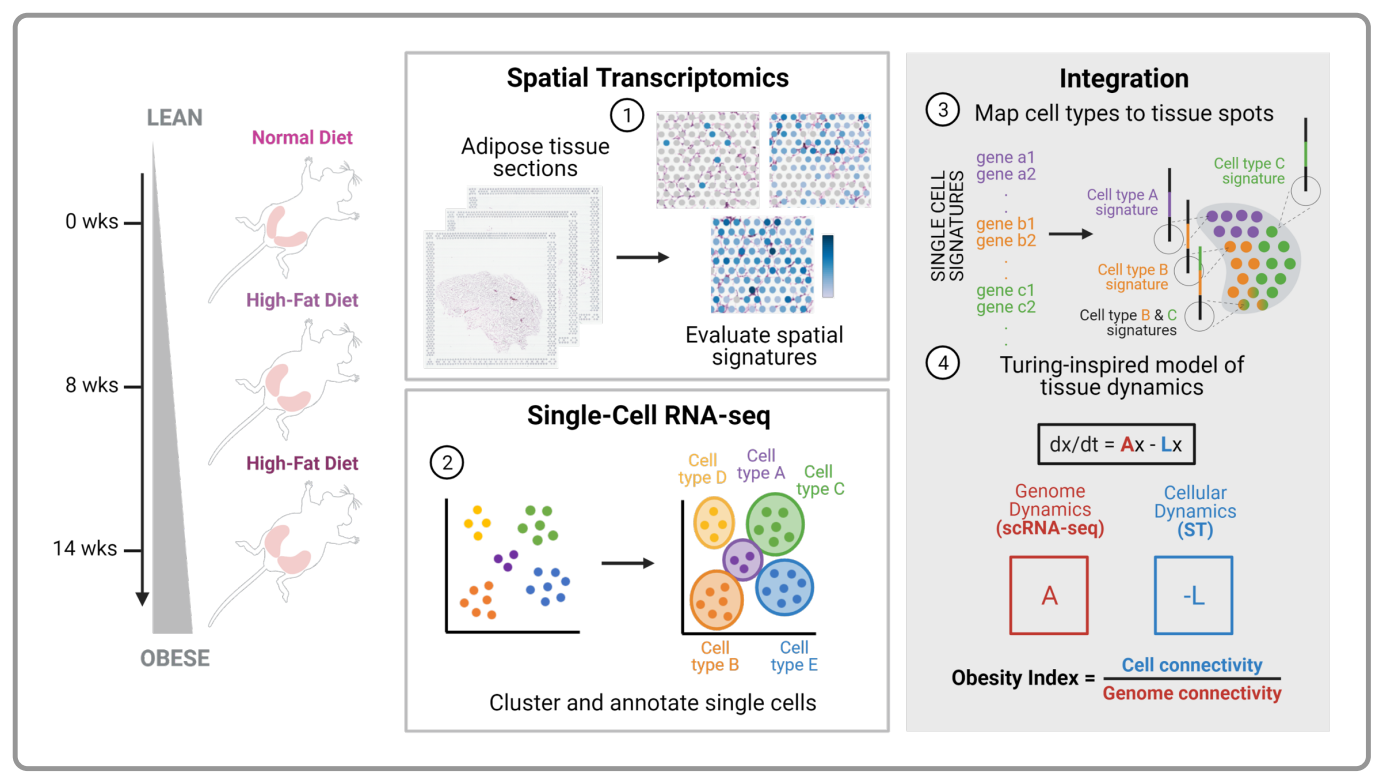

Figure 2. Workflow of individual and integrated analyses of spatial transcriptomics (ST) and single-cell RNA-sequencing (scRNA-seq) data. (1) First, adipose tissue sections from our three dietary conditions are evaluated for distribution of relevant marker genes. (2) Then, scRNA-seq data is grouped into transcriptionally similar clusters and cluster-specific signatures identified using a data-driven approach where we score markers for each cluster based on the marker's ubiquity, cluster-average expression, and uniqueness. We then use existing literature and databases to query these signatures and call cell types for each cluster. (3) Next, we map cell types to the spatial landscapes by finding an overlap between cell type signatures (from scRNA-seq) and tissue spot expression profiles (from ST). (4) Lastly, we model the disruption of adult adipose tissue morphogenesis and develop a metric for quantifying adipose tissue states by adapting the Turing system. 
As multiple nuclei can contribute transcripts to each spatial data spot, in parallel we performed single-cell RNA-seq on SVCs to clarify changes in immune cell types and facilitate signature identification in spatial data. For single-cell data, SVCs were isolated from fat pads contralateral to spatial data fat pads and enriched for $\mathrm{CD} 45^{+}$immune cells. scRNA-seq data were then aggregated across timepoints and spectrally clustered before ranking genes, establishing cluster-specific gene signatures (Table 1), and finally calling cell types for each cluster (Algorithm 1; Methods and Materials). Integrating our scRNA-seq and ST datasets, we mapped these cell types to tissue spots by finding where a cell type's signature and tissue spot's expression profile overlap (Algorithm 2; Methods and Materials). This tissue spot annotation step sets the stage for modeling the disruption of adult adipose tissue morphogenesis as a function of genome dynamics and cellular dynamics to ultimately quantify tissue states along the trajectory of obesity (Figure 2).

Algorithm 1: Clustering and Visualization

Input: Data matrix $\mathbf{X}_{m \times n}=\left(\mathbf{x}_{1}, \ldots, \mathbf{x}_{n}\right) \in \mathbb{R}^{m \times n}$ where $m$ rows are genes and $n$ columns are cells.

Output: Cell clusters and a low dimensional projection

1 Compute the sample mean $\mu_{n}$ and the centered matrix $\mathbf{X}_{c}=\mathbf{X}-\mu_{n} \mathbf{1}^{\top}$ where $\mathbf{1}$ is a vector of ones

2 Compute the SVD of $\mathbf{X}_{c}=\mathbf{U} \boldsymbol{\Sigma} \mathbf{V}^{\top}$

3 Construct $\mathbf{P}_{n \times r}=\left[\begin{array}{llll}v_{1} & v_{2} & \ldots & v_{r}\end{array}\right]$ where each column in $\mathbf{P}$ is a right singular vector of $\mathbf{X}_{c}$. Here $r$ can be chosen using the optimal hard threshold (Gavish and Donoho, 2014) on $\mathbf{X}_{c}$

${ }_{4}$ Construct a similarity matrix $\mathbf{A}_{n \times n}$ from $\mathbf{P}$ by determining the distance between each row. The choice of distance measure depends on the data type and user preference. Examples include Gaussian similarity, Euclidean distance, Manhattan distance (city block distance), Kullbeck-Liebler divergence, and correlation

5 Perform clustering: spectral or modularity clustering on $\mathbf{A}$ with $k$ clusters. $k$ can be chosen using domain knowledge or by testing multiple values of $k$ and evaluating the best performance. Note: $k$ may be $\leq r$

6 Visualization: t-SNE or UMAP to reduce the dimensions of $\mathbf{P}$ and visualize data colored according to clusters

Spectral clustering and data-guided cell type identification yielded six broad immune cell types: monocytes, T cells, ATMs, ATDCs, natural killer cells (NKCs), and B cells (Figure 3A, Figure S1). Changes in the cellular composition of adipose tissue in lean versus obese mice were largely driven by T cells, B cells, and ATMs, where the proportion of single cells classified as T cell and B cell decreased with obesity (17\% to $5 \%$ in T cell and $22 \%$ to $3 \%$ in B cell), while the proportion classified as ATMs sharply increased (43\% to $76 \%$ ). These findings are consistent with prior scRNA-seq studies of immune cell populations in diet-controlled mouse models (Jaitin et al., 2019; Weinstock et al., 2019), where the same panel of immune cells has been observed and where monocytes and ATMs have been the predominant populations present.

We next established localization patterns in lean and obese adipose tissue for these commonly characterized immune cell populations. To do so, we queried the cell type signatures across tissue spots at each timepoint, assigning cell types hierarchically based on the proportion of their signature expressed at the spot. In this way, the dominant cell type at a spot is the one with the highest number of its representative genes expressed (Figure 3B). These cell types assigned first at each tissue spot represent the most likely spot annotation and make up what we refer to as the 'dominant layer' (or Layer 1) of tissue spot assignments. The remaining cell types thereafter are successively assigned at each spot. Once this iterative assignment has been performed, we are left with a series of tissue assignment layers each representing a decreasing likelihood of a given 
cell type's presence across tissue spots (Figure S2). We refer to this process as spot deconvolution and the resulting tissue assignment layers as 'deconvoluted layers' (Algorithm 2; Methods and Materials).

Cell type localization in the dominant layer over time is consistent with trends in cellular composition from the scRNA-seq data where we observe a large but decreasing abundance of $T$ cells and $B$ cells and an emerging presence of ATMs in the HFD settings (Figure 3B). Adipocyte numbers and expansion are critical in the rearrangement of the adipose landscape during obesity as well, however, in this study, we emphasize the interplay between immune cell populations and their role in driving tissue function.

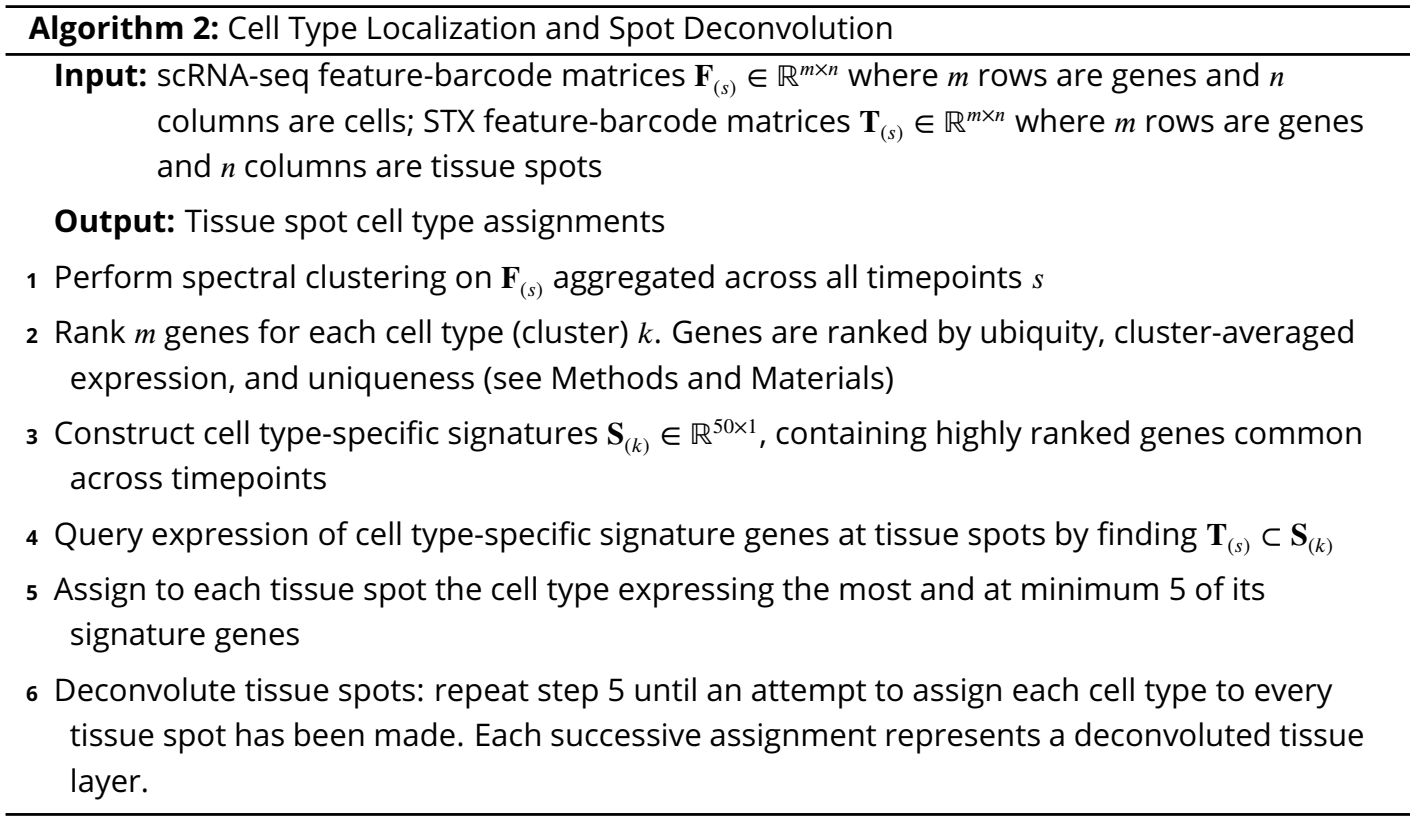

\section{Immune response and obesity}

Inflammation is a key contributor in adipose tissue dysfunction and development of insulin resistance in obesity. Cells of the adaptive and innate immune system both contribute to inflammatory processes in adipose tissue, including T cells, B cells, ATMs, ATDCs, and innate lymphoid cells (ILCS) (McLaughlin et al., 2017). This network of cells and their subtypes modulate inflammation through cytokine and chemokine secretion and cell-cell interactions, over time reducing antiinflammatory signaling and promoting proinflammatory cell types and processes. To broadly compare patterns of adaptive and innate immune cell networks in obesity, each tissue spot in the spatial data was classed as adaptive (T cell, B cell), innate (monocyte, NKCS, ATM, or ATDC), or mixed type based on the first two tissue assignment layers from Algorithm 2. We found that spots containing only adaptive immune cells consistently decreased with obesity, from $21 \%$ to $6 \%$ ( 3.5 fold change), while spots containing only innate immune cells increased with obesity, from 1\% to $9 \%$ ( 9 fold change) by 14w (Figure 3C), consistent with known shifts in cellularity with HFD feeding (Muir et al., 2018). Among total spots, $75 \%-85 \%$ had mixed type signatures, with higher numbers found in obesity. Overall, these data support a stronger influence of adipose tissue innate immune cells than adaptive immune cells with obesity, through increased quantity and frequency, strong spatial interspersion, and increased potential for cross-talk with the adaptive immune system (Figure 3D).

\section{Spatial patterning dynamics in adipose tissue}

Spatial transcriptomics data has supported clear delineations of different cell types present in known subregions of tissues, and profiling spatiotemporal patterns has helped define new anatom- 
bioRxiv preprint doi: https://doi.org/10.1101/2022.02.26.482134; this version posted February 27, 2022. The copyright holder for this preprint (which was not certified by peer review) is the author/funder. All rights reserved. No reuse allowed without permission.

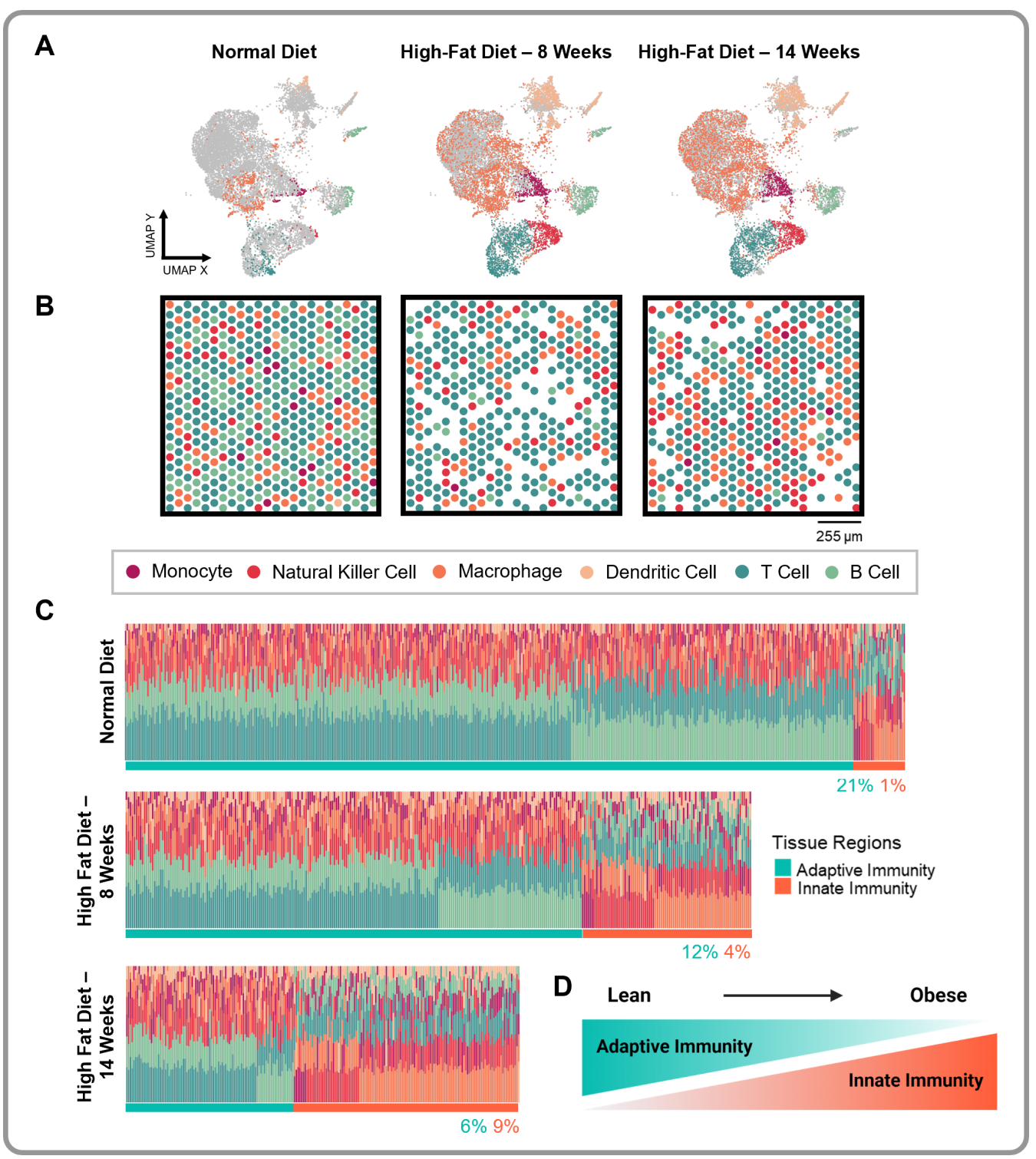

Figure 3. Immune cell type identification and localization in adipose tissue. (A) UMAP projections of scRNA-seq data over time, stratified by cell type. Gray points represent the UMAP projection from combined clustering of all three samples and colored points represent sample-specific single cells. (B) Annotated ST-derived tissue landscapes over time, zoomed in for clearer view of cell type composition and distribution. Cell type assignments represent the dominant spot assignments after performing spot deconvolution. White spaces with no spots indicate non-viable areas of the tissue where there were visible holes and therefore no detectable mRNA levels. (C) Stacked bar plots representing cell type proportions at a subset of tissue spots assigned to adaptive or innate immunity at each timepoint (denoted by orange and teal horizontal bars beneath plots). Tissue spots were classified as contributing to adaptive immunity if adaptive cell types colocalized at the spot, meaning deconvoluted tissue assignment layers 1 and 2 (L1 and L2) were assigned as $\mathrm{T}$ cell or B cell for those spots. Tissue spots were classified as contributing to innate immunity if innate cell types colocalized at the spot, meaning L1 and L2 were assigned as ATM, monocyte, NKC, or ATDC. Remaining tissue spots were classified as ambiguous tissue regions where L1 was assigned an adaptive cell type and L2 an innate cell type, or vice versa, and are not shown in the figure. Each stacked bar represents a single tissue spot. The height of each component in a stacked bar reflects cell type proportions (the number of genes expressed out of each 50-gene cell type-specific signature). (D) Model of adaptive and innate immunity trade-off during obesity progression. We propose that obesity weakens the adaptive immune system and innate immunity concurrently compensates for that weakened immune response over time. 


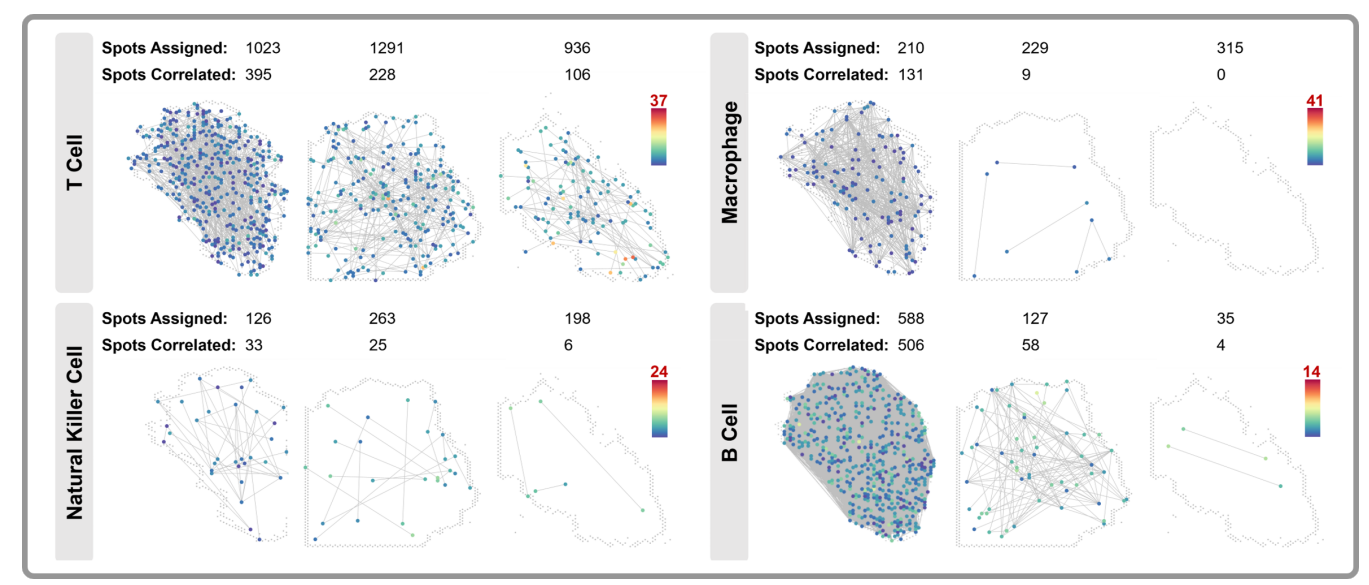

Figure 4. Immune cell type-specific spatial patterning. Network nodes represent transcriptionally correlated tissue spots assigned to the cell type in the dominant tissue assignment layer. Color intensity of network nodes represents the number of cell type-specific signature genes expressed at the spot. The color bar reflects the maximum number of signature genes (out of 50) expressed at any given spot across all three tissue settings. Network edges represent high correlation (correlation coefficient $\geq 0.85$ ) between cell type-specific gene expression profiles at two spots.

ical subregions and shed light on patterns that contribute to tissue dysfunction (Maniatis et al., 2019; Asp et al., 2019; Chen et al., 2020; Wallace et al., 2020; De Vries et al., 2020). Here we sought to better quantify patterns among the interspersed immune cell types in obese adipose tissue. In early diet-induced obesity in mice, proportional increases in ATMs relative to tissue weight and patterns of proliferation and apoptosis during weight gain and loss suggest active mechanisms of homeostatic signaling (Muir et al., 2018) that may eventually be overridden by chronic obesity (Zamarron et al., 2017). We therefore hypothesized that immune cell networks constructed from our time course data would capture the disrupted profiles of ATMs in obesity.

We constructed spatial cell networks for each major cell type, where nodes were the tissue spots assigned to the cell type based on the dominant layer and edges between nodes represented a correlation based on transcriptional signature. Surprisingly, cell type network edges decreased universally in obesity, indicating loss of transcriptional correlation among cells of the same type (Figure 4). Additionally, the distance over which the tissue spots correlated decreased, concurrent with increased heterogeneity in expression of cell type-specific signature genes. Loss of spatial patterning and increased heterogeneity support the emergence of immune cell subtypes in obesity that are not present in the lean state. Macrophages in particular show many phenotypic states depending on their environment (Suganami and Ogawa, 2010; Xue et al., 2014; Russo and Lumeng, 2018). Upon clustering macrophage-classified single cells into two sub-clusters, only one of the two clusters was represented in the normal diet setting while both were represented in the two highfat diet settings (Figure S3). The emergent cluster in the HFD settings was significantly enriched in $C d 9$ ( $\log _{2} \mathrm{FC}$ of 11.8 ), which has previously been observed in lipid-laden macrophages in obesity (Hill et al., 2018; Jaitin et al., 2019; Rondini and Granneman, 2020). In contrast to the observed macrophage subpopulations, T cell, B cell, and NKC sub-clusters were less distinct with fewer differentially expressed genes.

\section{Ligand-receptor colocalization}

To better understand specific interactions that might mediate shifted spatial patterning in obesity, we evaluated colocalization of paired ligands and receptors over time. We first captured ligand and receptor expression in adaptive and innate classes, characterizing four interaction states: (1) adaptive ligand and adaptive receptor, (2) innate ligand and innate receptor, (3) adaptive ligand and innate receptor, and (4) innate ligand and adaptive receptor (Methods and Materials). For 
bioRxiv preprint doi: https://doi.org/10.1101/2022.02.26.482134; this version posted February 27, 2022. The copyright holder for this preprint (which was not certified by peer review) is the author/funder. All rights reserved. No reuse allowed without permission.

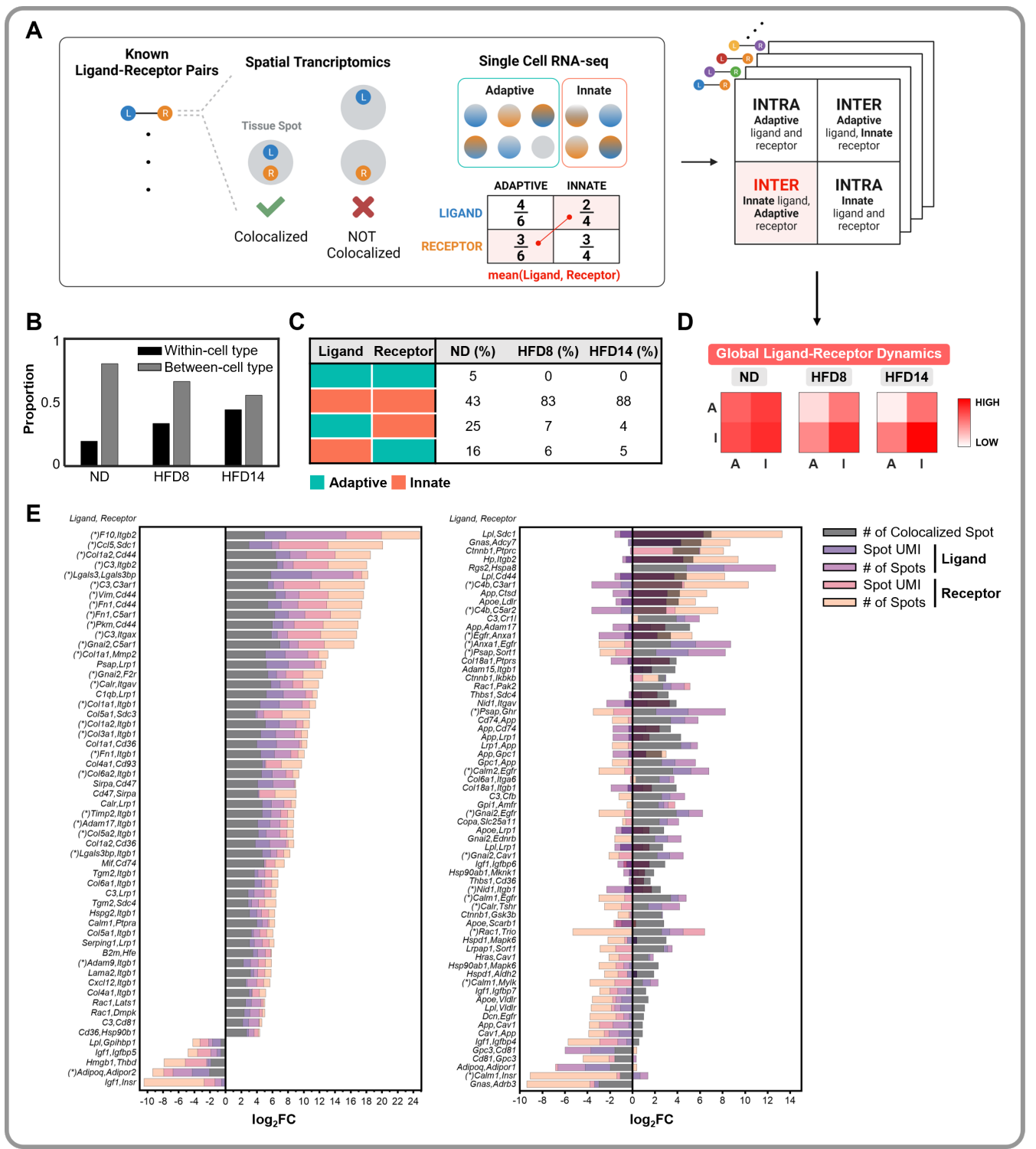

Figure 5. Cell-cell communication. (A) Illustration of ligand-receptor dynamics analysis workflow. For each known ligand-receptor pair (LR pair), each tissue spot in the spatial transcriptomics dataset is queried for co-expression $(\mathrm{UMI}>0$ ) of the ligand and receptor. Colocalized pairs are then further interrogated in the scRNA-seq dataset where the proportions of adaptive cell types expressing the ligand, adaptive cells types expressing the receptor, innate cell types expressing the ligand, and innate cell types expressing the receptor are evaluated, generating a 2-by-2 contingency table specific to the LR pair. Four interaction states are then defined based on these proportions by taking the mean between all pairwise combinations of differentially sourced ligands and receptors. For example, the score representing the inter-interaction between the illustrated innate ligand and adpative receptor (red shaded area in matrix) would be the average of $2 / 4$ and $3 / 6$ (taken from the contingency table), which is 0.5 . (B) Proportion of ligand-receptor interactions between single cells of the same cell type versus single cells of different cell types. (C) Percent of LR pairs that contributed preferentially (highest scores from interaction state matrices) to each interaction state across timepoints. (D) Global interaction state matrices across timepoints. Element-wise mean of LR pair-specific interaction state matrices output from workflow in (A). (E) Stacked bar plots represent LR pairs where the number of spots the pair colocalizes, expression level of $L$ and $R$ at the spot, and the number of spots separately expressing $L$ and $R$ all decreased or all increased from ND to HFD14 (left) or partially increased and partially decreased (right). 
this study, we determined transcript colocalization of known ligand-receptor pairs as a proxy for cell-cell communication given the restricted area of each tissue spot.

Interrogating known ligand-receptor pairs from the literature, we identified colocalized pairs in the ST data and then from the SCRNA-seq data, found the proportions of adaptive single cells expressing the pair's ligand and receptor, respectively, and the proportions of innate single cells expressing the pair's ligand and receptor, respectively (Figure 5A, Algorithm 3). This allows us to infer whether signaling interactions sourced from adaptive, innate, or an interplay of both contribute to the changes we see between lean and obese adipose tissue. We defined a score for each state by taking the mean proportion between all pairwise combinations of adaptive- or innate-sourced ligands and receptors (Figure 5A). Then for each colocalized ligand-receptor pair, we identified which of the four interaction states exhibited the highest score and found that the percentage of ligand-receptor pairs dominantly involved in adaptive ligand-adaptive receptor, adaptive ligandinnate receptor, and innate ligand-adaptive receptor signalling decreased during obesity progression while the percentage of pairs dominantly involved in innate ligand-innate receptor signaling increased (Figure 5C). Global interaction states - derived by taking the average of interaction state scores across all colocalized ligand-receptor pairs - reflected the same trend (Figure 5D). Based on scRNA-seq data, macrophages from obese versus lean adipose tissue increasingly accounted for the majority of ligand ( $85 \%$ vs. $44 \%$ ) and receptor ( $87 \%$ vs. $46 \%$ ) expression. Notably, B cells showed relatively strong contribution to ligand (22\%) and receptor (21\%) expression in lean mice, as did ATDCs in the $8 \mathrm{w}$ setting with $17 \%$ of ligand and $18 \%$ of receptor expression (Figure S4).

To further explore the contribution of different cell types to specific ligand-receptor interactions, we analyzed scRNA-seq data for clear expression patterns among colocalized ligand-receptor pairs. Interestingly, we found that there was a preference for ligands and receptors interacting from different cell types rather than the same cell type, though decreasingly so with obesity (Figure 5B). We then focused our search towards ligand-receptor pairs with high-confidence interactions (Methods and Materials). A summary of these pairs is shown in Figure 5E. We found that many of their ligand and receptor components were expressed from pairs of ATMs in both the ND (28\%) and HFD14 (58\%) settings. Lean adipose tissue was also enriched for ligand-receptor interactions between pairs of ATDCs and pairs of monocytes ( $25 \%$ for both). In contrast, obese adipose tissue also indicated frequent ligand-receptor interaction between monocytes and macrophage (13\%). We were primarily interested in determining the top colocalized ligand-receptor pairs that show an interaction between antigen presenting cells (ATMs, ATDCs) and T cells, but did not observe any.

\section{A Turing-inspired model for disruption of adult adipose tissue morphogenesis}

In 1952, Alan Turing famously described a simple two-cell system for modeling morphogenesis (Turing, 1952). At the time, the concept of a genome within a cell was not captured, but instead the reaction and diffusion of morphogens within and between cells was linearly represented. These two components or subnetworks that defined the relationship between the two cells were demonstrated to be mathematically stable, as evidenced by their respective negative eigenvalues. However, when combined, the resulting network contained a single positive eigenvalue, indicating the disruption of homeostasis in the system when the trade-off between within-cell and between-cell dynamics was considered (Box 1). In 2017, it was proposed that the coordinated effect of these two dynamical forces contribute to the emergence of function in tissue (Rajapakse and Smale, 2017). Here, we introduce the notion of the Turing system to our system of cells across tissue space. Like Turing, we aim to capture essential phenomena in our adapted model of emerging and morphing multicellular tissue function. For us, this means describing inter- and intra-cell type relationships at the cellular level and transcriptional diversity within cell type populations at the genome level. We achieve this by characterizing between-cell dynamics in terms of correlation between positionspecific gene expression profiles weighted by Euclidean distance from spatial transcriptomics data and characterizing within-cell dynamics in terms of correlation between single cells' gene expres- 


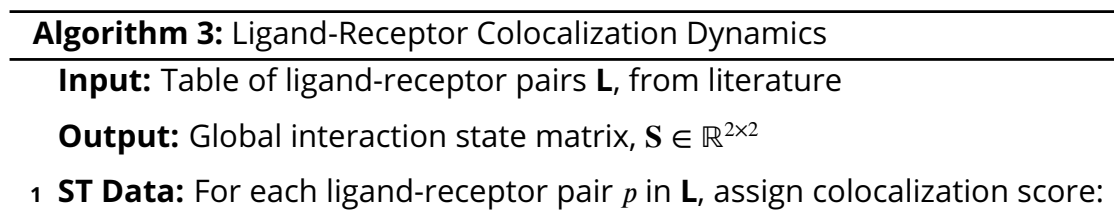

$$
c=\left\{\begin{array}{ll}
1 & \text { if } l>0 \text { and } r>0 \\
0 & \text { otherwise }
\end{array},\right.
$$

where $l$ and $r$ are UMI counts for the ligand and receptor in $p$, respectively, at a given tissue spot

2 scRNA-seq Data: If $p$ has a colocalization score of 1 , construct contingency table $\mathbf{C}_{2 \times 2}$, where $\mathbf{C}_{\mathbf{1 1}}$ and $\mathbf{C}_{\mathbf{2 1}}$ are the proportions of single cells classified as adaptive with $l$ and $r>3$, respectively, and $\mathbf{C}_{\mathbf{1 2}}$ and $\mathbf{C}_{\mathbf{2 2}}$ are the proportions of single cells classified as innate with $l$ and $r>3$, respectively

3 Construct local interaction state matrix for $\mathbf{S}_{(p)} \in \mathbb{R}^{2 \times 2}$, by finding the mean between all pairwise combinations of ligand-specific $\left(\mathbf{C}_{\mathbf{1 1}}, \mathbf{C}_{\mathbf{1 2}}\right)$ and receptor-specific $\left(\mathbf{C}_{\mathbf{2 1}}, \mathbf{C}_{\mathbf{2 2}}\right)$ proportions in $\mathbf{C}$

4 After performing steps 1-3 for all $p$ in $\mathbf{L}$, define global interaction state matrix, $\mathbf{S} \in \mathbb{R}^{2 \times 2}$, by computing the element-wise mean across all $\mathbf{S}_{(p)} \in \mathbb{R}^{2 \times 2}: \sum_{p=1}^{n} \mathbf{S}_{(p)} / n$

sion profiles from scRNA-seq data (Figure 6A, Methods and Materials). Taken together, we can comprehensively interrogate the emergence of tissue function in adipose tissue.

We started by characterizing emerging tissue function in terms of individual cell type contributions. To do so, we computed the tradeoff between cell and genome connectivity for each of the immune cell types present in our samples (Methods and Materials). This revealed an increasing contribution of the macrophage and monocyte populations across the tissue to the changing adipose landscape with obesity. Further consistent with the cell type localization trends we previously described, the contribution of T cells and B cells to emerging tissue function decreased with obesity (Figure 6B). To quantitatively discriminate between each tissue state, we developed an obesity index (OI), defined as the sum of the emergence of tissue function for each cell type normalized by the number of cell types represented in the tissue. We found that this index increases over time. Performing this analysis on our ND replicate further validated this trend where the OI was lower than both high-fat diet indices (data not shown). We next explored how each pair of cell types cooperatively contribute to tissue function, which we designate as the emergence of multicellular tissue function. To obtain this, we divide the emergence of local tissue function (cell and genome connectivity trade-off derived from merged data across two cell types) and emergence of global tissue function (cell and genome connectivity trade-off derived from merged data across all cell types).

With this approach, we captured inter- and intra- cell type connectivity and gained insight into functional coordination between cell types. We observed an overall decrease or stability in coupling with obesity among all cell type pairings, with the exception of the monocyte-macrophage axis which exhibited a sharp increase in coupling with obesity (Figure 6C). As monocytes are known to differentiate into mature resident macropahge (van Furth and Cohn, 1968), this positive cooperative relationship is not surprising. Their cooperation being one of few cell type relationships to contribute highly to tissue function in the obese state is noteworthy however. In the small intestine, macrophages rely on replenishment from monocytes to maintain intestinal homeostasis, as they do not divide and therefore cannot undergo self-renewal (Bain and Mowat, 2014). Our modeling demonstrates a slight uptick in monocyte contribution to tissue function (Figure 6B) lending plausibility to the pooling of those extra resources to further support resident macrophage 


\section{Box 1. Turing's model of pattern formation}

Alan Turing's contribution to mathematical biology produced remarkable insights into the formation of patterns, like animal stripes and spots or limb development Turing (1952). Turing described the state of a biological system in terms of the concentrations and "diffusibilities" of its substances - referred to as "morphogens." The mathematical formulation of Turing's model for morphogenesis relies on a set of differential equations - reaction-diffusion equations Maini (2012).

Turing postulated that discontinuous diffusion drives a system to become unstable and establish spatial patterns due to a breakdown in symmetry and homogeneity. Turing's two-cell system contains variables $\mathbf{x}_{1}, \mathbf{y}_{1}$ representing concentrations of molecules (or proteins) for the first cell and $\mathbf{x}_{2}, \mathbf{y}_{2}$ for the second cell:

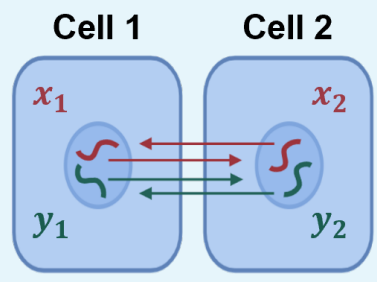

Turing's two-cell reaction-diffusion equations are given as:

$$
\begin{aligned}
& \dot{\mathbf{x}}_{1}=\left(5 \mathbf{x}_{1}-6 \mathbf{y}_{1}+1\right)+0.5\left(\mathbf{x}_{2}-\mathbf{x}_{1}\right) \\
& \dot{\mathbf{y}}_{1}=\left(6 \mathbf{x}_{1}-7 \mathbf{y}_{1}+1\right)+4.5\left(\mathbf{y}_{2}-\mathbf{y}_{1}\right) \\
& \dot{\mathbf{x}}_{2}=\left(5 \mathbf{x}_{2}-6 \mathbf{y}_{2}+1\right)+0.5\left(\mathbf{x}_{1}-\mathbf{x}_{2}\right) \\
& \dot{\mathbf{y}}_{2}=\underbrace{\left(6 \mathbf{x}_{2}-7 \mathbf{y}_{2}+1\right)}_{\begin{array}{c}
\text { Reaction (A) } \\
\text { (within-cell) }
\end{array}}+\underbrace{4.5\left(\mathbf{y}_{1}-\mathbf{y}_{2}\right)}_{\begin{array}{c}
\text { Diffusion (-L) } \\
\text { (between-cell) }
\end{array}}
\end{aligned}
$$

where, $\dot{\mathbf{x}}=\frac{d \mathbf{x}}{d t}, \dot{\mathbf{y}}=\frac{d \mathbf{y}}{d t}$ and $\mathbf{x}, \mathbf{y}>0$. We can rewrite Equation 1 as follows:

$$
\begin{aligned}
&\left(\begin{array}{l}
\dot{\mathbf{x}}_{1} \\
\dot{\mathbf{y}}_{1} \\
\dot{\mathbf{x}}_{2} \\
\dot{\mathbf{y}}_{2}
\end{array}\right)=\left(\begin{array}{cccc}
5 & -6 & 0 & 0 \\
6 & -7 & 0 & 0 \\
0 & 0 & 5 & -6 \\
0 & 0 & 6 & -7
\end{array}\right)\left(\begin{array}{l}
\mathbf{x}_{1} \\
\mathbf{y}_{1} \\
\mathbf{x}_{2} \\
\mathbf{y}_{2}
\end{array}\right)+\left(\begin{array}{cccc}
-0.5 & 0 & 0.5 & 0 \\
0 & -4.5 & 0 & 4.5 \\
0.5 & 0 & -0.5 & 0 \\
0 & 4.5 & 0 & -4.5
\end{array}\right)\left(\begin{array}{l}
\mathbf{x}_{1} \\
\mathbf{y}_{1} \\
\mathbf{x}_{2} \\
\mathbf{y}_{2}
\end{array}\right) \\
&=\left(\begin{array}{cccc}
4.5 & -6 & 0.5 & 0 \\
6 & -11.5 & 0 & 4.5 \\
0.5 & 0 & 4.5 & -6 \\
0 & 4.5 & 6 & -11.5
\end{array}\right)\left(\begin{array}{l}
\mathbf{x}_{1} \\
\mathbf{y}_{1} \\
\mathbf{x}_{2} \\
\mathbf{y}_{2}
\end{array}\right)
\end{aligned}
$$

Separately, the reaction and diffusion dynamics of the system are stabilizing (Equation 2), as their eigenvalues are negative $\left(\lambda_{i}(\mathbf{A})=-1, i=1,2,3,4\right.$ and $\lambda_{i}(-\mathbf{L})=-9,-1,0,0, i=1,2,3,4$, respectively). The combined system has one positive and three negative eigenvalues $\left(\lambda_{i}=\right.$ $1,1,14, i=1,2,3,4)$, rendering the system unstable (Equation 3 ). Turing's beautifully designed two-cell system demonstrated that only with diffusion is the system unstable - an instability needed for the formation of pattern (Rajapakse and Smale, 2017; Chua, 2005). We can mathematically represent this system in the form $\frac{d \mathbf{x}}{d t}=\mathbf{A x}-\mathbf{L x}, \mathbf{x}=\left(\mathbf{x}_{1}, \ldots, \mathbf{x}_{n}\right)$. 


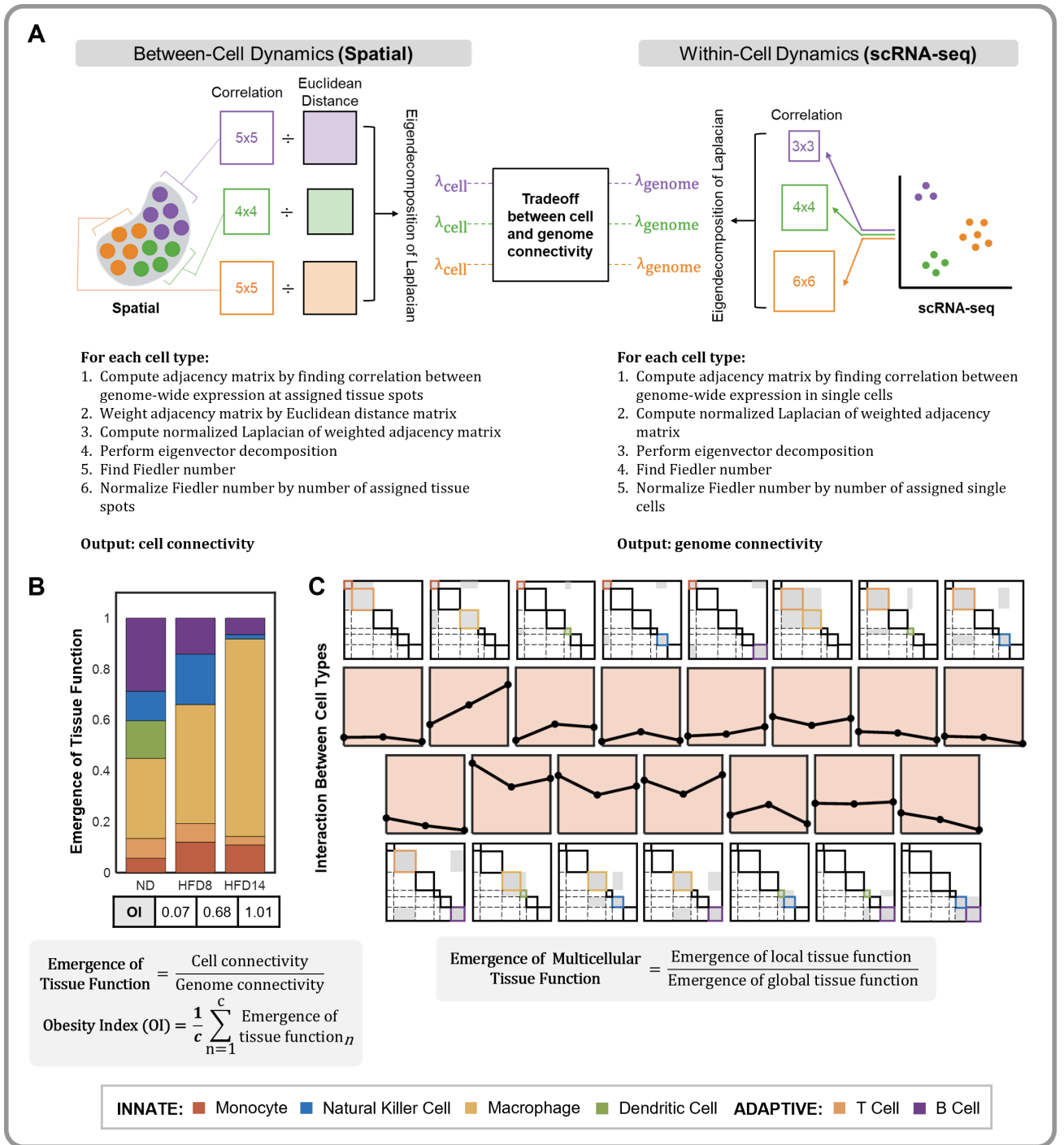

Figure 6. Emergence of tissue function in lean versus obese adipose. (A) Overview of Turing-inspired model for describing adult adipose tissue dynamics. (B) Individual cell type contributions to tissue function (normalized to sum to 1) and corresponding table of obesity indices (quantitative summary of tissue state) over time. Parameter ' $c$ ' in the Ol equation represents the number of cell types in the tissue. (C) Pairwise relationship between cell types over time. The outer perimeter of matrices with pairs of colored squares along the diagonal highlight the two cell types being compared in the light orange-shaded plots directly below or above. The gray-shaded regions of these matrices represent the inter- and intra-cell type connectivity between the two cell types that reflects the emergence of multicellular tissue function plotted in the center shaded panels. We compute emergence of multicellular tissue function by dividing the the 'emergence of local tissue function' - the tradeoff between cell and genome connectivity for two cell types - by the 'emergence of global tissue function - the tradeoff between cell and genome connectivity for all cell types'. 
renewal or in some way activate non-resident and/or self-renewing macrophage populations in obese adipose tissue. In the context of intestinal homeostasis versus inflammatory bowel disease, different differentiation outcomes can give rise to resident or inflammatory macrophage, respectively (Bain et alo, 2013). It is possible that this phenomena is paralleled here, where the growth of the monocyte-macrophage axis might suggest that in the inflammatory environment of obese adipose tissue, a bifurcation in the monocyte differentiation trajectory occurs to further promote an obesogenic state. We also found that macrophage pairings - particularly with other innate cell types - largely demonstrated high though decreasing coupling with other lineages over the course of obesity progression. Additionally, the T cell-B cell axis exhibits one of the lowest cooperative contributions, consistent with a weakening adaptive immune response.

\section{Discussion}

Inflammation is a prominent feature of obese adipose tissue identified as a contributor to development of insulin resistance. Here, investigation of immune cell signatures in their spatial context highlights the tissue-wide scale of innate and myeloid cell population expansion.

An important caveat to this work is that the spatial transcriptomics platform, barcoded spots are $55 \mu \mathrm{m}$ in diameter with a $100 \mu \mathrm{m}$ center-to-center distance, with the potential for multiple nuclei at each spot that scales depending on the thickness of a section. While the sensitivity and resolution of our scRNA-seq dataset enabled us to more precisely infer cell identities across the tissue, exploring sequencing-based spatial transcriptomic platforms with smaller spot diameters in following iterations of this work will be valuable (Cho et al., 2021).

The collection of spatial transcriptomic data for this study served as a pilot test for extended exploration of tissue landscapes and obesity to come, so in an effort to prioritize feasibility and cost, we only collected a data replicate for the normal diet sample. Our following research in this area will include more data replicates to facilitate further validation of our findings.

One common challenge in distinguishing between cell types, especially among related lineages, is the overlap between core signature genes. Notably, dendritic cells and macropahge express similar marker genes. To circumvent this, we developed a data-guided approach for systematically parsing the transcriptome-wide profile of genes in our data to find those highly representative and unique within each cluster of cells. The final panel of markers we used to define cell types (Table 1) contains some genes that are consequently lesser known in the literature but that were nonetheless cluster-specific and represented in both the single-cell and spatial datasets. The robustness of this approach was demonstrated by the consensus between our called cell types and those called by the reputable ImmGen database using our data as input. Our approach further allowed us to define phenotypes of these known cell types specific to our samples based on observed enriched and depleted genes.

This study provides a proof-of-concept for predicting the trajectory of tissue states during disease. Moving forward, our model of tissue morphogenesis disruption can be generalized for any disease context, where more broadly, we can define a disease index capable of distinguishing between healthy and unhealthy tissue states. Liver and intestinal tissue, for example, are pathologically relevant to this study as they too play a role in the development of obesity. In the small intestine, genes involved in lipid metabolism and metabolic homeostasis are affected in an obese state (De Wit et al., 2008, 2011) while in liver tissue, obesity correlates with a higher presence of Th17-related cytokines (Cavallari et al., 2016). Performing integrated analysis of spatial and singlecell transcriptomics on these tissues could allow us to establish a spatially-aware, inter-tissue network for obesity while testing the robustness of our model. The next step in evaluating how well our model distinguishes between tissue samples with different pathologies will be validating our findings using classified tissue biopsies as ground truth.

Spatial pattering in adipose tissue indicated that cell types may adopt alternate phenotypes during the course of obesity onset. Notably, we observed a $C d 9$-rich macrophage subpopulation 
emerge in obese adipose tissue, though emerging subpopualations from the other cell types were less identifiable in our data. Earlier studies have demonstrated, however, that $\mathrm{Cd} 8^{+} \mathrm{T}$ effector and $\mathrm{Cd}^{+} \mathrm{T}_{H} 1$ cell numbers rise while regulatory $\mathrm{T}$ cell numbers fall with obesity (Reilly and Saltiel, 2017). Additionally, visceral adipose tissue in mice have been found to harbor an increased number of IgG+ $B$ cells during obesity (Winer et al., 2011). The only differentially expressed gene observed in our B cell sub-clustering was the immunoglobulin gene, Ighg3, which was enriched in the high-fat diet settings ( $\log _{2} \mathrm{FC}$ of 1 ) so while we observed B cell numbers decrease writ large, our data suggests that the remaining population is largely comprised of a known pathogenic class of $B$ cells in obesity. This pattern supports a potential role for adaptive immune cells in driving obesity, wherein a new class of adaptive immune cells promoting rather than protecting against the obesity phenotype begins to emerge. Thus, while the recruitment of adaptive immune cells to fight inflammation in adipose tissue is disrupted, adaptive immunity is not completely futile in an obese state. These insights could be pivotal in considering how we might target immune signatures in treating or preventing obesity.

Signaling between cells is critical to tissue development, and consequently the disruption of a tissue's normal course of development (Nelson, 2009). Analysis of ligand-receptor activity in lean versus obese adipose tissue revealed a striking trend. Using co-expression at the same tissue position as a proxy for co-localization, we found that ligands and receptors expressed in innate cell types were preferentially co-localized across the obese tissue compared to ligands and receptors expressed in adaptive cell types or a combination of both. This further supports the role that the innate immune system plays in promoting an obese state. Future work will undoubtedly be needed to validate the presence of these ligand-receptor interactions as well as capture longer range crosstalk in the tissue coming from endocrine or paracrine signaling. Remarkably, however, fitting our spatial and single-cell data to an adapted form of Turing's system modeling within-cell and between-cell dynamics captured the same trend wherein innate cell types increasingly contributed to tissue function with obesity (Figure 7).

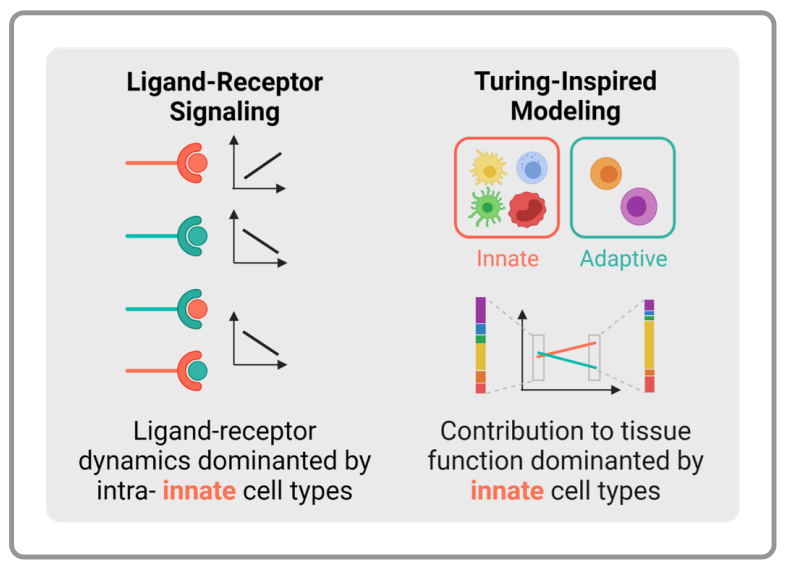

Figure 7. Consensus between ligand-receptor signaling analysis and Turing-inspired modeling.

Cancer is a known co-morbidity of obesity (Calle et al., 2003; Gallagher and LeRoith, 2015). More recently, it was shown that obesity-induced accumulation of IL-6-producing pro-inflammatory metabolically activated macrophage in mammary adipose tissue promotes triple-negative breast cancer (Tiwari et al., 2019). The relationship between obesity and cancer is easily discernible one of the primary hallmarks of obesity is inflammation and inflammation promotes tumor formation and stemness properties in cancer cells (Grivennikov et al., 2010). The mechanisms by which obesity influences different cancers however is not fully understood. Leveraging spatial assays like spatial transcriptomics and characterizing tissue dynamics during obesity can enable better 
understanding of how obesity influences increased cancer risk.

Obesity can cause prolonged changes to the adipose cellular landscape and persistent inflammatory phenotypes like insulin resistance and macrophage proliferation (Zamarron et al., 2017). In this study, we have gained a clearer understanding of the changing tissue architecture during obesity, bringing us closer to accurate prognoses, treatments, and being able to reprogram diseased tissue (Srivastava and DeWitt, 2016).

\section{Methods and Materials}

\section{Animals}

C57BL/6J mice were used for these experiments (Jackson Laboratories 000664). Male mice were fed ad libitum a control normal chow diet (ND; 13.4\% fat, 5L0D LabDiet) or high-fat diet (HFD; 60\% calories from fat, Research Diets D12492) for the indicated amount of time starting at 9 weeks old. Animals were housed in a specific pathogen-free facility with a $12 \mathrm{~h}$ light/12 h dark cycle and given free access to food and water except for temporary fasting associated with glucose tolerance tests. All mouse procedures were approved by the Institutional Animal Care and Use Committee (IACUC) at the University of Michigan (Animal Welfare Assurance Number D16-00072 (A3114-01)), and in compliance with the Institute of Laboratory Animal Research Guide for the Care and Use of Laboratory Animals.

\section{Glucose tolerance tests}

For glucose tolerance tests (GTT), starting four hours into the light cycle, mice were fasted with ad libitum access to water for six hours in clean cages. A 100 mg/mL D-glucose (Sigma G7021) solution was prepared in sterile -/- DPBS and injected at $0.7 \mathrm{~g} / \mathrm{kg}$ of body weight. Area under the curve (AUC) calculations were performed using the log trapezoidal method.

\section{Stromal cell isolation}

Stromal vascular cells (SVCs) were collected from adipose tissues as in (Ref Muir 2018 Rapid Adipose). After cardiac perfusion, adipose tissues were collected, minced finely to $3-5 \mathrm{~mm}$ pieces, and added to ice cold $\mathrm{HBSS}+\mathrm{Ca} / \mathrm{Mg}$ (Insert CO). Up to $1.5 \mathrm{~g}$ of tissue per sample was digested in $10 \mathrm{ml}$ of $1 \mathrm{mg} / \mathrm{mL}$ collagenase II (Sigma C68850) in $\mathrm{HBSS}+\mathrm{Ca} / \mathrm{Mg}$ at $37^{\circ} \mathrm{C}$ for 45 minutes with vigorous shaking. Digests were filtered through buffer-soaked 100 micron cell strainers and centrifuged at $300 \times \mathrm{g}$ at $4 \mathrm{C}$ to pellet SVCs.

\section{Immune cell enrichment}

SVCs were enriched for CD45+ immune cells using Biolegend MojoSort Mouse CD45 Nanobeads (Biolegend 480027), following the manufacturer's protocol. Briefly, SVC pellets were resuspended in $1 \mathrm{~mL}$ MojoSort Buffer, pooling the four samples from each cohort into a single respective cohort tube (ND, 8w, 14w), then filtered through a 70 micron cell strainer and placed in $5 \mathrm{~mL}$ polypropylene tubes. After addition of nanobeads, samples were sequentially processed for magnetic separation. To increase purity, three magnetic separations in total were performed on the labeled fractions. Final cell suspensions were filtered through 40 micron pipette tip filters.

\section{Antibody barcoding and single-cell RNA-sequencing}

TotalSeqB anti-mouse antibodies (Biolegend) were used for protein barcoding prior to single cell RNA-sequencing, using the manufacturer's recommended protocol. Cell viability was $>80 \%$ with $<15 \%$ aggregation. Cells were Fc blocked on ice using CD16/32 antibody, then stained in Biolegend staining buffer with $1 \mathrm{uL}$ of Biolegend TotalSeqB antibody per sample including CD3 (100257), CD4 (100573), CD19 (115563), F4/80 (123155), CD11C (117359), Mac-2 (125425), CD161 (108763), and CD11b (101273). 


\section{Spatial transcriptomics tissue preparation}

Within 30 minutes of cardiac perfusion, adipose tissues were pre-soaked in ice cold O.C.T. Compound (VWR 25608-930) and placed in biopsy cryomolds (VWR 25608-922) with fresh O.C.T., rapidly frozen by immersion in liquid nitrogen-cooled isopentane, and kept on dry ice or at $-80^{\circ} \mathrm{C}$ until sectioning. Fresh tissue sections were cut at $10 \mu \mathrm{m}$ after 20 minute equilibration in a cryochamber set to $-26^{\circ} \mathrm{C}$ or below with specimen arm at $-40^{\circ} \mathrm{C}$. Samples were placed on a $10 \mathrm{X}$ Genomics/Visium Spatial Gene Expression slide and processed by the University of Michigan Advanced Genomics Core according to the manufacturer's protocol.

\section{Spatial transcriptomics data processing}

Raw sequencing data were processed using the 10X Genomics SpaceRanger (1.0.0) pipeline with mouse reference $\mathrm{GRCm} 38$, and resulting feature-barcode matrices were loaded into MATLAB for further processing using an in-house pipeline. Matrices contain absolute transcript counts, reported as unique molecular identifier (UMI) counts, pertaining to each feature (rows) and tissueassociated barcode (columns). Informed by the 10X Genomics Loupe Browser visualization of barcoded tissue spots overlaying tissue histology images, we filtered out barcoded spots that overlapped visible holes in the tissue and therefore had no tissue material for gene expression to be detected at. This resulted in no barcoded spots being discarded from from the ND setting, 1,458 being discarded from the HFD8 setting, and 490 from the HFD14 setting. After filtering, there were $2,035,1,936$, and 1,504 viable spots remaining in each setting, respectively. Genes that were detected at no barcoded tissue spots (indicated by a row sum of 0 ) were filtered from the featurebarcode matrices. Additionally, we filtered out mitochondrial and ribosomal genes as well as genes known to be linked to mapping errors (Malat, Lars2, Kcnq10t1, and Gm42418) (van den Brink et al., 2020). Our working features list contained 19,941 total genes. The median number of expressed genes per tissue spot for each setting was: 91,105 , and 173, respectively. Finally, we normalized each feature-barcode matrix by dividing by a scaling factor - computed as the median UMI counts per barcode divided by the total UMI counts per barcode - then log-transforming the matrix.

\section{Single-cell RNA-sequencing data processing}

Raw single-cell RNA-sequencing data were processed using the 10X Genomics CellRanger pipeline and resulting feature-barcode matrices were loaded into MATLAB for further processing using an in-house pipeline. Single cells (barcodes) with fewer than 500 expressed genes were filtered from the data matrix leaving 1,231 cells in the ND setting, 6,011 cells in the HFD8 setting, and 6,258 cells in the HFD14 setting. Genes (features) were filtered out according to the same criteria used to filter genes in the spatial transcriptomics data. The median number of genes expressed per cell for each setting was: 1,707, 1,644, and 1583, respectively. Additionally, filtered matrices were normalized in the same way as the spatial transcriptomics data matrices.

\section{Integration of spatial and single-cell transcriptomics data}

The cellular composition of each tissue section was characterized by mapping expression profiles from scRNA-seq data to their paired spatial landscape. To achieve this, we first aggregated our scRNA-seq data matrices, mean-centered and dimensionally reduced the resulting matrix to the first 20 singular vectors, then performed spectral clustering using Euclidean distance as the metric for generating the similarity graph. Informed by known cell types found in adipose tissue from literature, we selected for 15 clusters $(k=15)$. We then employed a data-guided cell type identification approach where we scored markers for each cluster based on ubiquity (percent of cells in the cluster expressing each gene), cluster-averaged expression (average expression of each gene among all cells in the cluster), and uniqueness (see 'Marker Uniqueness' subsection). For each cluster, the top 50 genes with the highest cumulative score that were also expressed across all three dietary settings and expressed in the spatial transcriptomics data, were selected as clusterspecific signatures. We used prior literature and the CellMarker database (Zhang et al., 2019) to 
query these signatures and call cell types for each cluster. At this point, we further narrowed our set of cluster by removing two clusters indicative of fibroblast contamination, removing two clusters of unknown lineage whose signatures contained non-essential genes, and merging clusters with redundant cell type classifications, leaving us with six total clusters. We validated our cell type annotations using Immgen and found that they aligned with Immgen signatures (Heng et al., 2008). To annotate our spatial transcriptomics tissue sections, we mapped cell types identified from the scRNA-seq data to tissue spots, based on the proportion of signature genes expressed at each spot (see 'Spot Deconvolution' subsection).

\section{Marker uniqueness}

To evaluate the uniqueness of potential cell type markers to a cluster, we found the ratio of the total number of clusters overexpressing a given gene (fold change $\geq 2$ ) to the number of times that gene is overexpressed by the cluster of interest. The resulting value increases a marker's score proportional to how frequently we see the gene expressed highly in one cluster compared to all other clusters, but offsets the score if the gene is highly expressed in multiple clusters. Ultimately, non-unique genes rank low with this approach. We found that this approach is similar to the Term Frequency-Inverse Document Frequency (TF-IDF) technique commonly applied in machine learning contexts to determine the importance of a word within a document or set of documents (Salton and Buckley, 1988). For proof-of-concept, we adapted the TF-IDF technique for marker ranking by treating documents as clusters and words as genes, where term frequency corresponded to the number of times a given gene is overexpressed by the cluster of interest and inverse document frequency to the number of clusters overexpressing the gene. In doing so, we yielded similar results (data not shown).

\section{Spot deconvolution}

Given the thickness of tissue sections and diameter of tissue spots, it is likely that two or more cell identities will colocalize at the same tissue spot. To deconvolute each spot into their possible cellular components, we start by defining a dominant tissue layer wherein a cell type is assigned to a spot if at least five of the cell type-specific signature genes are expressed at the spot and a higher proportion of its signature is expressed at the spot than any other cell type. We define subsequent tissue layers by assigning remaining cell types to each tissue spot following the same criteria as long as the cell type has not already been assigned to the spot in a previous layer. Additionally, we impose a tiebreak condition for when two or more cell types express the same number of genes at a spot. When this happens, the tied cell types are assigned according to highest to lowest cumulative expression of their expressed signature genes. With this approach, each cell type has an equal likelihood of being assigned to a layer, giving us a total number of layers equivalent to the number of cell types identified in the tissue.

\section{Ligand-receptor analysis}

Known ligand-receptor pairs in mice were taken from a compendium of pairs reported throughout the literature compiled by the Lewis Lab at UCSD. (Armingol et al., 2021). For each ligand-receptor pair and timepoint, we scanned the tissue space to identify tissue spots where both the ligand and receptor of the pair colocalize. We define colocalization as the spatial overlap or co-expression of the ligand and receptor (UMI count $>0$ ) belonging to a known pair at the same $x-, y$-coordinate (tissue spot) in the spatial transcriptomics data. Paired ligands and receptors that colocalized at at least one tissue spot were further queried for cell-type specific expression at the single-cell level, where the independent expression of the ligand and receptor in adaptive and innate immunity cells was evaluated. The proportions of single cells annotated as: (1) adaptive immunity cell types that expressed the ligand, (2) adaptive immunity cell types expressing the receptor, (3) innate immunity cell types expressing the ligand, and (4) innate immunity cell types expressing the receptor were captured in a 2-by-2 contingency table. The UMI count cutoff for ligand or receptor expression 
was 3. Then, a local interaction state matrix was constructed capturing for a given ligand-receptor pair the intra-interaction between the ligand and receptor in adaptive cells (mean of proportions 1 and 2), the intra-interaction between the ligand and receptor in innate cells (mean of proportions 2 and 3), the inter-interaction between the ligand in adaptive and receptor in innate cells (mean of proportions 1 and 4), and the inter-interaction between the ligand in innate cells and receptor in adaptive cells (mean of proportions 3 and 2). Finally, a global interaction state matrix summarizing these intra- and inter-interactions among all colocalized ligand-receptor pairs was derived by taking the element-wise mean across the local state tables.

To highlight the most significant ligand-receptor pairs active in our data, we identified those that are most likely to interact, using the following criteria: colocalization at 5 or more tissue spots in the spatial data, expression in the scRNA-seq data with a UMI > 3, and no significantly higher expression in adipocytes than stromal vascualar cells. Additionally the ligand and recptor had to independently be expressed in at least $10 \%$ more of a given cell type population than in any other population.

\section{Modeling tissue function}

While our spatial transcriptomics data is not single-cell resolution, we are able to assign a dominant cell type to each tissue spot based on the expression of cell type-specific signatures. We can then treat each tissue spot as an individual cell in our model of emerging tissue function. While we also described non-dominant layers of cell type assignments at each spot, we focus our model on the dominant cell type assignment. Our model draws on two subnetworks of the overall tissue network - the cell-cell network and within-cell genome network. Taken together, we can represent each cell type's individual contribution to tissue function and define an index describing the tissue's state in terms of cellular and genome connectivity.

\section{Cellular connectivity}

Spatial transcriptomics captures the expression profiles of cells tissue-wide in Euclidean space, allowing us to observe patterns of between-cell connectivity across the tissue. We start by characterizing intra-cell type relationships within individual cell populations across the tissue (emergence of tissue function) by finding the transcriptome-wide correlation between tissue spots assigned to a given cell type. We then weight each pairwise correlation coefficient by the Euclidean distance between their corresponding tissue spots, generating an adjacency matrix. We next perform eigenvector decomposition on the normalized Laplacian of the adjacency matrix to obtain the Fiedler number, representing the connectivity of the network of transcriptionally similar cells across the tissue - cell connectivity. The cell type-specific Fiedler number is then divided by the number of tissue spots assigned to the cell type to normalize for the variability in network sizes across the tissue.

To capture inter-cell type contributions to tissue function (emergence of multicellular tissue function) we consider a network of networks where each cell type is a subnetwork within the larger tissue network. We repeat our previous steps, this time generating an adjacency matrix based on the correlation in expression between tissue spots from two cell types instead of one. We do this for every pair of cell types represented in our tissue samples to demonstrate how cell type coupling contributes to tissue function.

\section{Genome connectivity}

From single-cell RNA-seq, we compare the diversity in gene expression profiles within a given cell type to capture within-cell genome connectivity across the tissue. Similar to our computation for cellular connectivity, we find the transcriptome-wide correlation between single cells classified as a given cell type. We then perform eigenvector decomposition on the normalized Laplacian of this correlation matrix to obtain the Fiedler number which we use to define genome connectivity. 
Again, we normalize the Fiedler number, this time dividing by the number of single cells assigned to the cell type.

\section{Acknowledgments}

We thank Ingrid Bergin and Pavlina Zafirovska for technical assistance in sectioning and staining tissues, Olivia Koues and the University of Michigan Advanced Genomics Core, Evan Keller and Greg Shelley for assistance with single cell sequencing, Alnawaz Rehemtulla for critical feedback and support of the murine studies, Abigail Riesmeyer and Siva Kumar for technical assistance, Stephen Lindsly and Can Chen for their input on data analysis, and Cooper Stansbury for image analysis. We would also like to acknowledge BioRender.com which was used to generate Figures 2, 5A and 7. Research reported in this publication was supported by the University of Michigan NIH NIGMS Bioinformatics Training Grant under Award Number 5T32GM070449-15 and the University of Michigan Genome Science Training Program (GSTP) Fellowship funded by NHGRI under Award Number 5T32HG000040-27 to GAD, the Air Force Office of Scientific Research (AFOSR) award FA9550-18-10028 to IR, the National Institute Of Diabetes And Digestive And Kidney Diseases of the National Institutes of Health under Award Number K01DK116928 to LAM, and the National Cancer Institute of the National Institutes of Health under Award Number P30CA046592 by the use of the Rogel Cancer Center Single Cell Resource. The content is solely the responsibility of the authors and does not necessarily represent the official views of the National Institutes of Health.

\section{References}

Armingol E, Officer A, Harismendy O, Lewis NE. Deciphering cell-cell interactions and communication from gene expression. Nature Reviews Genetics. 2021; 22(2):71-88.

Asp M, Giacomello S, Larsson L, Wu C, Fürth D, Qian X, Wärdell E, Custodio J, Reimegård J, Salmén F, et al. A spatiotemporal organ-wide gene expression and cell atlas of the developing human heart. Cell. 2019; 179(7):1647-1660.

Bäckdahl J, Franzén L, Massier L, Li Q, Jalkanen J, Gao H, Andersson A, Bhalla N, Thorell A, Rydén M, et al. Spatial mapping reveals human adipocyte subpopulations with distinct sensitivities to insulin. Cell metabolism. 2021; 33(9):1869-1882.

Bain CC, Mowat AM. The monocyte-macrophage axis in the intestine. Cellular immunology. 2014; 291(1-2):4148.

Bain C, Scott C, Uronen-Hansson H, Gudjonsson S, Jansson O, Grip O, Guilliams M, Malissen B, Agace WW, Mowat AM. Resident and pro-inflammatory macrophages in the colon represent alternative contextdependent fates of the same Ly6C hi monocyte precursors. Mucosal immunology. 2013; 6(3):498-510.

van den Brink SC, Alemany A, van Batenburg V, Moris N, Blotenburg M, Vivié J, Baillie-Johnson P, Nichols J, Sonnen KF, Arias AM, et al. Single-cell and spatial transcriptomics reveal somitogenesis in gastruloids. Nature. 2020; 582(7812):405-409.

Calle EE, Rodriguez C, Walker-Thurmond K, Thun MJ. Overweight, obesity, and mortality from cancer in a prospectively studied cohort of US adults. New England Journal of Medicine. 2003; 348(17):1625-1638.

Cavallari JF, Denou E, Foley KP, Khan WI, Schertzer JD. Different Th17 immunity in gut, liver, and adipose tissues during obesity: the role of diet, genetics, and microbes. Gut Microbes. 2016; 7(1):82-89.

Chen WT, Lu A, Craessaerts K, Pavie B, Frigerio CS, Corthout N, Qian X, Laláková J, Kühnemund M, Voytyuk I, et al. Spatial transcriptomics and in situ sequencing to study Alzheimer's disease. Cell. 2020; 182(4):976-991.

Cho CS, Xi J, Si Y, Park SR, Hsu JE, Kim M, Jun G, Kang HM, Lee JH. Microscopic examination of spatial transcriptome using Seq-Scope. Cell. 2021; .

Chua LO. Local activity is the origin of complexity. International journal of bifurcation and chaos. 2005; 15(11):3435-3456. 
Dai H, Alsalhe TA, Chalghaf N, Riccò M, Bragazzi NL, Wu J. The global burden of disease attributable to high body mass index in 195 countries and territories, 1990-2017: An analysis of the Global Burden of Disease Study. PLOS Medicine. 2020 07; 17(7):1-19. https://doi.org/10.1371/journal.pmed.1003198, doi: 10.1371/journal.pmed.1003198.

De Vries NL, Mahfouz A, Koning F, De Miranda NF. Unraveling the complexity of the cancer microenvironment with multidimensional genomic and cytometric technologies. Frontiers in oncology. 2020; p. 1254.

De Wit NJ, Boekschoten MV, Bachmair EM, Hooiveld GJ, de Groot PJ, Rubio-Aliaga I, Daniel H, Müller M. Dosedependent effects of dietary fat on development of obesity in relation to intestinal differential gene expression in C57BL/6J mice. PLoS One. 2011; 6(4):e19145.

De Wit NJ, Bosch-Vermeulen H, de Groot PJ, Hooiveld GJ, Bromhaar MMG, Jansen J, Müller M, van der Meer $\mathrm{R}$. The role of the small intestine in the development of dietary fat-induced obesity and insulin resistance in C57BL/6J mice. BMC medical genomics. 2008; 1(1):1-16.

van Furth R, Cohn ZA. The origin and kinetics of mononuclear phagocytes. The Journal of experimental medicine. 1968; 128(3):415-435.

Gallagher EJ, LeRoith D. Obesity and diabetes: the increased risk of cancer and cancer-related mortality. Physiological reviews. 2015; 95(3):727-748.

Gavish M, Donoho DL. The optimal hard threshold for singular values is $4 / \sqrt{3}$. IEEE Transactions on Information Theory. 2014; 60(8):5040 - 5053.

Grivennikov SI, Greten FR, Karin M. Immunity, inflammation, and cancer. Cell. 2010; 140(6):883-899.

Heng TS, Painter MW, Elpek K, Lukacs-Kornek V, Mauermann N, Turley SJ, Koller D, Kim FS, Wagers AJ, Asinovski $\mathrm{N}$, et al. The Immunological Genome Project: networks of gene expression in immune cells. Nature immunology. 2008; 9(10):1091-1094.

Hill DA, Lim HW, Kim YH, Ho WY, Foong YH, Nelson VL, Nguyen HC, Chegireddy K, Kim J, Habertheuer A, et al. Distinct macrophage populations direct inflammatory versus physiological changes in adipose tissue. Proceedings of the National Academy of Sciences. 2018; 115(22):E5096-E5105.

Jaitin DA, Adlung L, Thaiss CA, Weiner A, Li B, Descamps H, Lundgren P, Bleriot C, Liu Z, Deczkowska A, et al. Lipid-associated macrophages control metabolic homeostasis in a Trem2-dependent manner. Cell. 2019; 178(3):686-698.

Liu C, Li P, Li H, Wang S, Ding L, Wang H, Ye H, Jin Y, Hou J, Fang X, et al. TREM2 regulates obesity-induced insulin resistance via adipose tissue remodeling in mice of high-fat feeding. Journal of Translational Medicine. 2019; 17(1):1-11.

Lumeng CN, DelProposto JB, Westcott DJ, Saltiel AR. Phenotypic switching of adipose tissue macrophages with obesity is generated by spatiotemporal differences in macrophage subtypes. Diabetes. 2008; 57(12):32393246.

Lumeng CN, DeYoung SM, Bodzin JL, Saltiel AR. Increased inflammatory properties of adipose tissue macrophages recruited during diet-induced obesity. Diabetes. 2007; 56(1):16-23.

Maini PK. Turing's mathematical theory of morphogenesis. . 2012; .

Maniatis S, Äijö T, Vickovic S, Braine C, Kang K, Mollbrink A, Fagegaltier D, Žaneta Andrusivová, Saarenpää S, Saiz-Castro G, Cuevas M, Watters A, Lundeberg J, Bonneau R, Phatnani H. Spatiotemporal dynamics of molecular pathology in amyotrophic lateral sclerosis. Science. 2019; 364(6435):89-93. https://www. science. org/doi/abs/10.1126/science.aav9776, doi: 10.1126/science.aav9776.

McLaughlin T, Ackerman SE, Shen L, Engleman E, et al. Role of innate and adaptive immunity in obesityassociated metabolic disease. The Journal of clinical investigation. 2017; 127(1):5-13.

Misumi I, Starmer J, Uchimura T, Beck MA, Magnuson T, Whitmire JK. Obesity Expands a Distinct Population of $\mathrm{T}$ Cells in Adipose Tissue and Increases Vulnerability to Infection. Cell Reports. 2019; 27(2):514-524.e5. https://www.sciencedirect.com/science/article/pii/S2211124719303468, doi: https://doi.org/10.1016/j.celrep.2019.03.030. 
${ }_{652}$ Morris DL, Cho KW, DelProposto JL, Oatmen KE, Geletka LM, Martinez-Santibanez G, Singer K, Lumeng CN.
653 Adipose tissue macrophages function as antigen-presenting cells and regulate adipose tissue CD4+ T cells 654 in mice. Diabetes. 2013; 62(8):2762-2772.

655

Muir LA, Cho KW, Geletka LM, Baker NA, Flesher CG, Ehlers AP, Kaciroti N, Lindsly S, Ronquist S, Rajapakse I, et al. Human CD206+ macrophages associate with diabetes and adipose tissue lymphoid clusters. JCI insight. 2022; .

Muir LA, Kiridena S, Griffin C, DelProposto JB, Geletka L, Martinez-Santibañez G, Zamarron BF, Lucas H, Singer K, O'Rourke RW, et al. Frontline Science: Rapid adipose tissue expansion triggers unique proliferation and lipid accumulation profiles in adipose tissue macrophages. Journal of leukocyte biology. 2018; 103(4):615-628.

Nelson CM. Geometric control of tissue morphogenesis. Biochimica et Biophysica Acta (BBA)-Molecular Cell Research. 2009; 1793(5):903-910.

Porsche CE, Delproposto JB, Geletka L, O’Rourke R, Lumeng CN. Obesity results in adipose tissue T cell exhaustion. $\mathrm{JCl}$ insight. $2021 ; 6(8)$.

Porsche CE, Delproposto JB, Patrick E, Zamarron BF, Lumeng CN. Adipose tissue dendritic cell signals are required to maintain T cell homeostasis and obesity-induced expansion. Molecular and cellular endocrinology. 2020; 505:110740.

Rajapakse I, Smale S. Emergence of function from coordinated cells in a tissue. Proceedings of the National Academy of Sciences. 2017; 114(7):1462-1467.

Reilly SM, Saltiel AR. Adapting to obesity with adipose tissue inflammation. Nature Reviews Endocrinology. 2017; 13(11):633-643.

Rondini EA, Granneman JG. Single cell approaches to address adipose tissue stromal cell heterogeneity. Biochemical Journal. 2020; 477(3):583-600.

Russo L, Lumeng CN. Properties and functions of adipose tissue macrophages in obesity. Immunology. 2018; 155(4):407-417.

Salton G, Buckley C. Term-weighting approaches in automatic text retrieval. Information processing \& management. 1988; 24(5):513-523.

Sharif O, Brunner JS, Korosec A, Martins R, Jais A, Snijder B, Vogel A, Caldera M, Hladik A, Lakovits K, et al. Beneficial metabolic effects of TREM2 in obesity are uncoupled from its expression on macrophages. Diabetes. 2021; 70(9):2042-2057.

Srivastava D, DeWitt N. In vivo cellular reprogramming: the next generation. Cell. 2016; 166(6):1386-1396.

StåhI PL, Salmén F, Vickovic S, Lundmark A, Navarro JF, Magnusson J, Giacomello S, Asp M, Westholm JO, Huss $\mathrm{M}$, et al. Visualization and analysis of gene expression in tissue sections by spatial transcriptomics. Science. 2016; 353(6294):78-82.

Suganami T, Ogawa Y. Adipose tissue macrophages: their role in adipose tissue remodeling. Journal of leukocyte biology. 2010; 88(1):33-39.

Tiwari P, Blank A, Cui C, Schoenfelt KQ, Zhou G, Xu Y, Khramtsova G, Olopade F, Shah AM, Khan SA, et al. Metabolically activated adipose tissue macrophages link obesity to triple-negative breast cancer. Journal of Experimental Medicine. 2019; 216(6):1345-1358.

Turing A. The chemical basis of morphogenesis. Philos Trans Roy Soc. 1952; .

Uysal KT, Wiesbrock SM, Marino MW, Hotamisligil GS. Protection from obesity-induced insulin resistance in mice lacking TNF- $\alpha$ function. Nature. 1997; 389(6651):610-614.

Vijay J, Gauthier MF, Biswell RL, Louiselle DA, Johnston JJ, Cheung WA, Belden B, Pramatarova A, Biertho L, Gibson M, et al. Single-cell analysis of human adipose tissue identifies depot-and disease-specific cell types. Nature metabolism. 2020; 2(1):97-109.

Wallace ML, Huang KW, Hochbaum D, Hyun M, Radeljic G, Sabatini BL. Anatomical and single-cell transcriptional profiling of the murine habenular complex. Elife. 2020; 9:e51271. 
712

713

714

Weinstock A, Brown EJ, Garabedian ML, Pena S, Sharma M, Lafaille J, Moore KJ, Fisher EA. Single-cell RNA sequencing of visceral adipose tissue leukocytes reveals that caloric restriction following obesity promotes the accumulation of a distinct macrophage population with features of phagocytic cells. Immunometabolism. 2019; 1.

Weisberg SP, McCann D, Desai M, Rosenbaum M, Leibel RL, Ferrante AW, et al. Obesity is associated with macrophage accumulation in adipose tissue. The Journal of clinical investigation. 2003; 112(12):1796-1808.

Winer DA, Winer S, Shen L, Wadia PP, Yantha J, Paltser G, Tsui H, Wu P, Davidson MG, Alonso MN, et al. B cells promote insulin resistance through modulation of T cells and production of pathogenic IgG antibodies. Nature medicine. 2011; 17(5):610-617.

Xue J, Schmidt SV, Sander J, Draffehn A, Krebs W, Quester I, De Nardo D, Gohel TD, Emde M, Schmidleithner $L$, et al. Transcriptome-based network analysis reveals a spectrum model of human macrophage activation. Immunity. 2014; 40(2):274-288.

Zamarron BF, Mergian TA, Cho KW, Martinez-Santibanez G, Luan D, Singer K, DelProposto JL, Geletka LM, Muir LA, Lumeng CN. Macrophage proliferation sustains adipose tissue inflammation in formerly obese mice. Diabetes. 2017; 66(2):392-406.

Zhang X, Lan Y, Xu J, Quan F, Zhao E, Deng C, Luo T, Xu L, Liao G, Yan M, et al. CellMarker: a manually curated resource of cell markers in human and mouse. Nucleic acids research. 2019; 47(D1):D721-D728. 


\begin{tabular}{|c|c|c|c|c|c|}
\hline Monocyte & T Cell & Macrophage & Dendritic Cell & $\begin{array}{c}\text { Natural Killer } \\
\text { Cell }\end{array}$ & B Cell \\
\hline Gngt2 & $117 r$ & $C 1 q b$ & Ppt1 & $\mathrm{Ccl} 5$ & $\lg k c$ \\
\hline Msrb1 & Thy 1 & C1qa & $C d 24 a$ & $A W 112010$ & $C d 79 b$ \\
\hline Clec4a3 & $\mathrm{H} 2-\mathrm{Q7}$ & $\mathrm{Clqc}$ & Naaa & Ugcg & $\mathrm{H} 2-\mathrm{DMb2}$ \\
\hline Clec4a1 & Ets1 & Mrc1 & Batf3 & $H 2-Q 7$ & Ebf1 \\
\hline Csf1r & Ptprcap & Csf1r & Psmb9 & Ptprcap & $\operatorname{lghm}$ \\
\hline$G s r$ & $L t b$ & $D a b 2$ & Naga & Ctla2a & Plac8 \\
\hline Plac8 & Malat1 & Trem2 & Wdfy4 & Klrk1 & $H 2-E b 1$ \\
\hline$C e b p b$ & $T m s b 4 x$ & Pf4 & Fnbp1 & Gimap6 & Mef2c \\
\hline Rnf149 & Rac2 & Adgre1 & $B C 028528$ & $H 2-Q 6$ & $\mathrm{H} 2-\mathrm{Aa}$ \\
\hline$C y b b$ & Gata3 & C3ar1 & $\operatorname{lrf8}$ & Gimap5 & $H 2-A b 1$ \\
\hline Mafb & Gimap6 & C5ar1 & Cbfa2t3 & Ets1 & $C d 74$ \\
\hline$C d 300 a$ & Tpt1 & Fcgr3 & Ppm1m & Tyrobp & Cd83 \\
\hline Fcgr3 & Eef1a1 & Lilr4b & Rgs10 & Fcer1g & Serp1 \\
\hline Emilin2 & Fau & Ehd4 & Aif1 & $1 d 2$ & $C d 37$ \\
\hline$M s 4 a 6 c$ & $A c t b$ & Pltp & Rnase6 & Rac2 & Gimap6 \\
\hline$T p d 52$ & $H 2-K 1$ & Slc11a1 & Xcr1 & Cd52 & Pou2f2 \\
\hline Pla2g7 & Cd52 & Glul & $I d 2$ & Ctsd & Ly6e \\
\hline Ncf2 & $H 2-D 1$ & Cd84 & Psme1 & Malat1 & Foxp1 \\
\hline$L y z 2$ & Tmsb10 & Lgmn & Lsp1 & $T m s b 4 x$ & Ralgps2 \\
\hline S100a4 & $U b b$ & Slc6a6 & Syngr2 & Lgals1 & $L y 6 a$ \\
\hline $\operatorname{Prdx5}$ & $\operatorname{Actg} 1$ & Mafb & $H 2-D M b 1$ & Ptprc & Trp53inp1 \\
\hline Ifitm3 & Coro1a & Abhd12 & Plbd1 & $A c t b$ & Fam $214 a$ \\
\hline $\mathrm{Ccl} 6$ & $B 2 m$ & $\mathrm{Cc} / 9$ & Anpep & Arhgdib & Ptprcap \\
\hline Alox5ap & $P f n 1$ & $C y b b$ & $H 2-A a$ & Eef1a1 & Fam46c \\
\hline $\mathrm{Cc} / 9$ & Hspa8 & Apoe & Tap1 & Tpt1 & Ets1 \\
\hline Sirpa & Eif1 & Daglb & $H 2-E b 1$ & Tmsb10 & Shisa5 \\
\hline Apoe & Ppia & Cd14 & $R t / 8 b$ & $\mathrm{H} 2-\mathrm{K} 1$ & Scd1 \\
\hline $\mathrm{Hck}$ & $H 2-Q 6$ & Lipa & Unc119 & Fau & D10Wsu102e \\
\hline Plbd1 & $5100 a 4$ & $C d 36$ & $R a b 7 b$ & $H 2-D 1$ & Malat1 \\
\hline$T g f b i$ & Fth1 & Mt1 & Cadm1 & $\operatorname{Actg} 1$ & Capg \\
\hline Pou2f2 & Rack1 & $L y z 2$ & Rab32 & Corola & Phgdh \\
\hline Zeb2 & $H 3 f 3 b$ & Pld3 & $\mathrm{H} 2 a f z$ & Pfn1 & Dennd4a \\
\hline Cxcr4 & Uba52 & Tbxas1 & $R t / 8 a$ & Dok2 & Srgn \\
\hline Lilr $4 b$ & Srgn & Ctsd & Atpif1 & $B 2 m$ & Cacnale \\
\hline Metrnl & Ptprc & Sirpa & $H 2-A b 1$ & Ppia & $T m s b 4 x$ \\
\hline Lgals3 & Eef2 & Tmem106a & Sh3bp1 & $U b b$ & Cd52 \\
\hline Ifi27/2a & Sh3bgr/3 & $S d c 3$ & Mpeg1 & $C f / 1$ & Tpt1 \\
\hline Tyrobp & $C f / 1$ & Blvrb & Olfm1 & Myl6 & Fau \\
\hline Mpeg1 & Ftl1 & $C d 63$ & Kctd12 & Sh3bgrl3 & Eef1a1 \\
\hline Plin2 & Hsp90ab1 & Timp2 & Plekho1 & Eif1 & Tnfrsf13b \\
\hline Fcer1g & Ptma & Atp6v1b2 & Actr3 & $\mathrm{Cd} 37$ & Unc93b1 \\
\hline Srgn & Ccnd2 & Selenop & Atox1 & Cond2 & Man1a \\
\hline Ctss & Gm10076 & $L p l$ & $\mathrm{Cd} 74$ & Laptm5 & St6gal1 \\
\hline Nfe2/2 & Myl6 & Dhrs3 & Psme2 & $H 3 f 3 b$ & $A c t b$ \\
\hline$W f d c 17$ & $D d x 5$ & Ccl6 & Gltp & Uba52 & Pkig \\
\hline Cd68 & Laptm5 & Zeb2 & Psmb8 & Ikzf3 & Fth1 \\
\hline Lamp1 & Junb & $H 2-A a$ & $L y z 2$ & Anxa2 & $1 k z f 3$ \\
\hline Lyn & Oaz1 & Capg & $C k b$ & $B c / 2$ & $H 3 f 3 b$ \\
\hline Taldo1 & Jund & Ctsl & Pomp & Lcp1 & Eif1 \\
\hline Sat1 & S100a10 & $H 2-E b 1$ & Alox5ap & Rack1 & Ctss \\
\hline
\end{tabular}

Table 1. Cell-type signatures. 50 highest scoring genes per cell type. 


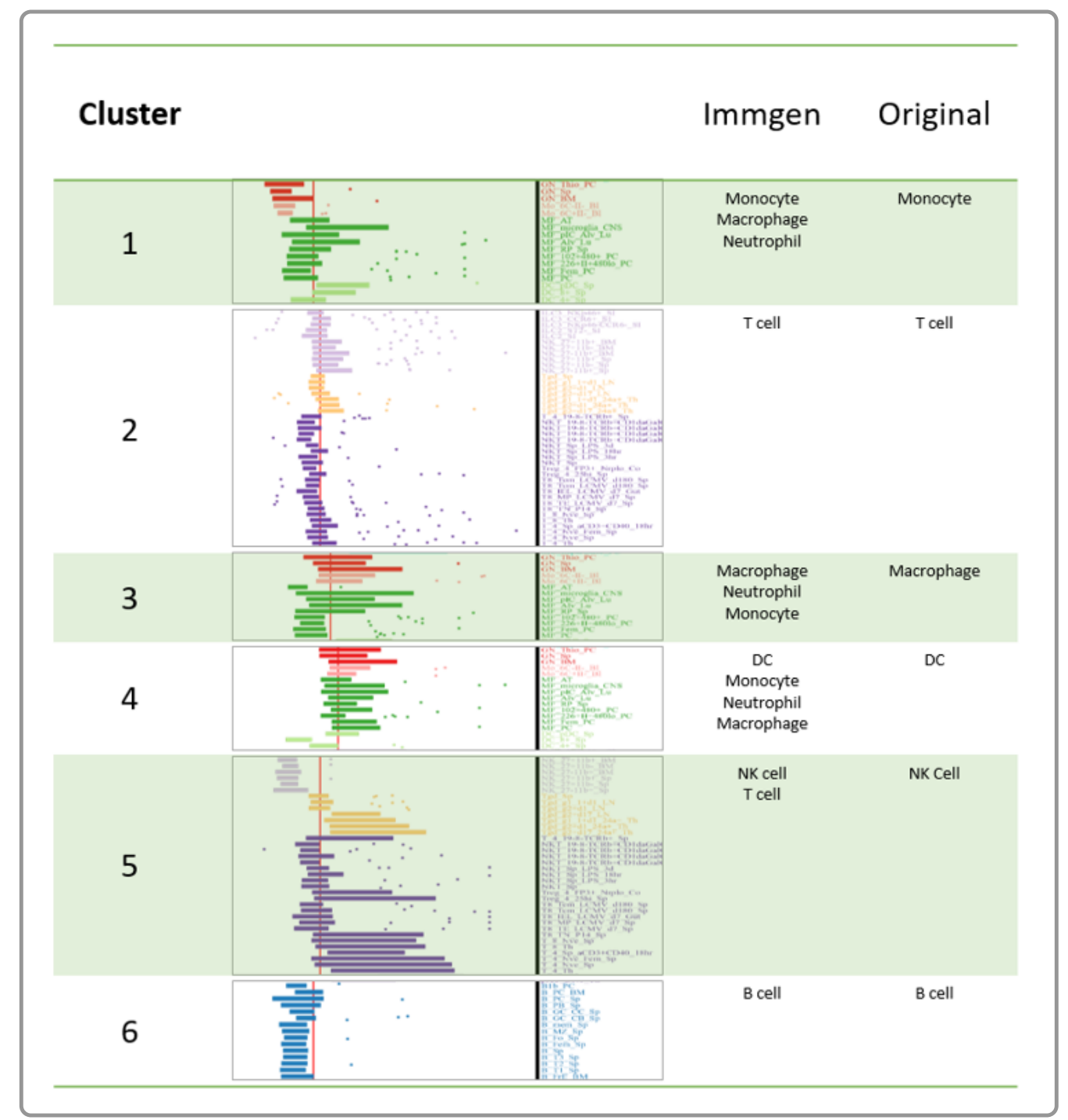

Supplementary Figure 1. Validation of clustering and cell type annotation. Cell types in the single-cell data align with Immgen signatures. 
bioRxiv preprint doi: https://doi.org/10.1101/2022.02.26.482134; this version posted February 27, 2022. The copyright holder for this preprint (which was not certified by peer review) is the author/funder. All rights reserved. No reuse allowed without permission.

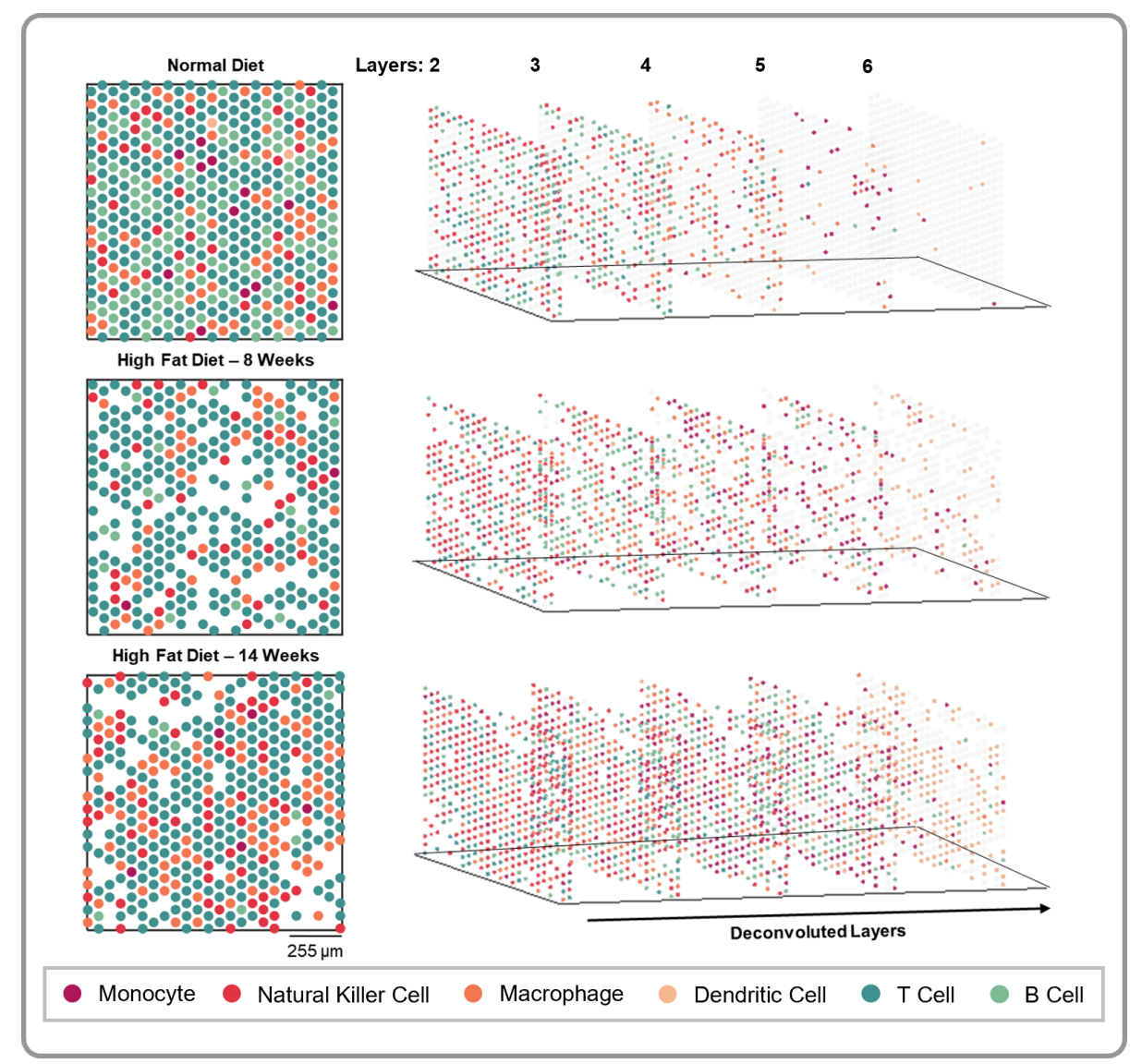

Supplementary Figure 2. Deconvoluted tissue assignment layers. Annotated spatial transcriptomics tissue landscapes over time, zoomed in for clearer view of cell type composition and distribution. Cell type assignments in the left panels represent the dominant spot assignments after performing spot deconvolution (i.e. Layer 1). (Rightmost panels) Non-dominant deconvoluted layers (Layers 2 - 6) of tissue spot assignments for zoomed in region. Light gray spots represent spots that were unassigned because no remaining cell types met the gene expression threshold. 


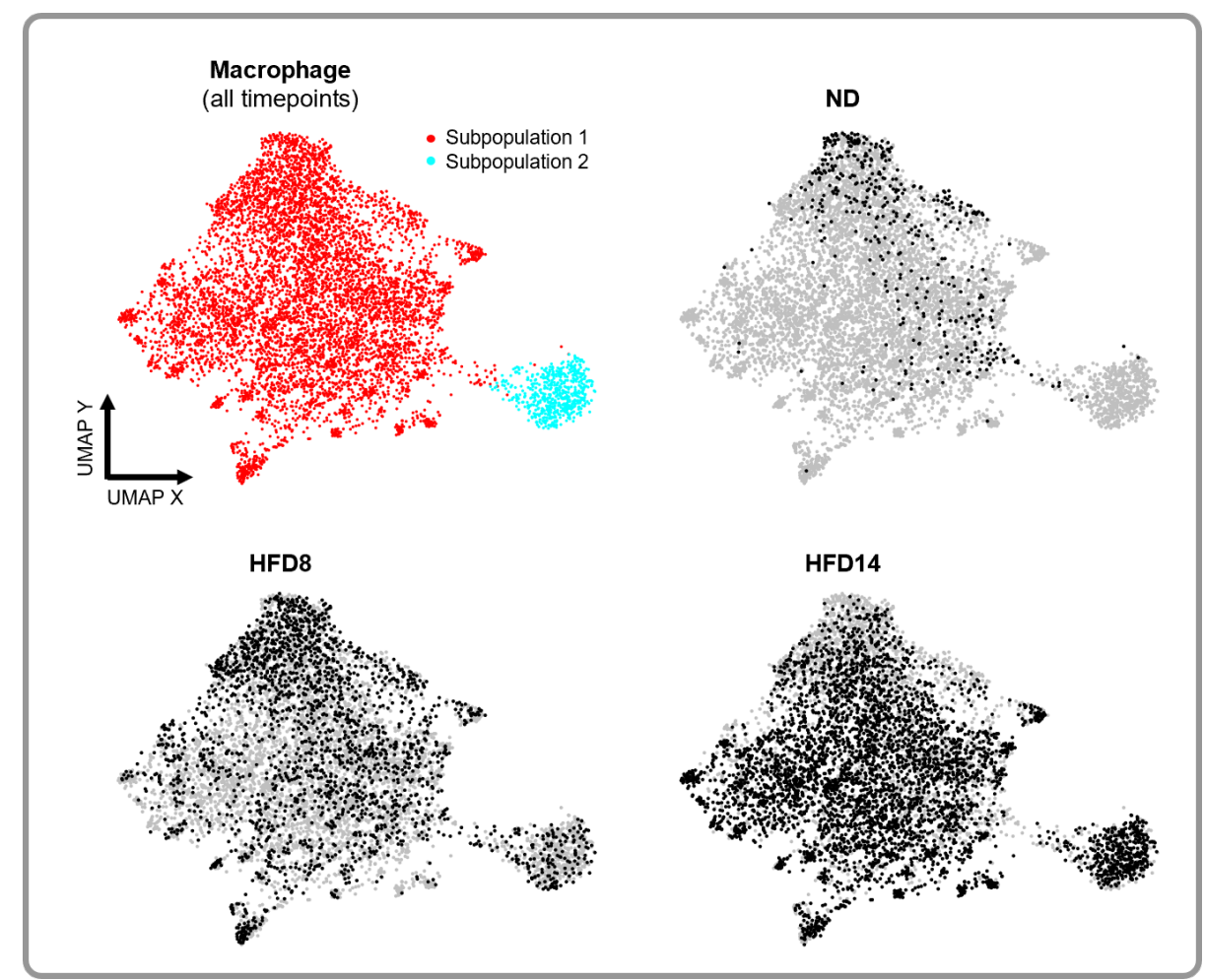

Supplementary Figure 3. Spectral clustering on single cells assigned macrophage. Subpopulation 2 is significantly enriched for $C d 9$, a gene known to be highly expressed in obesity.

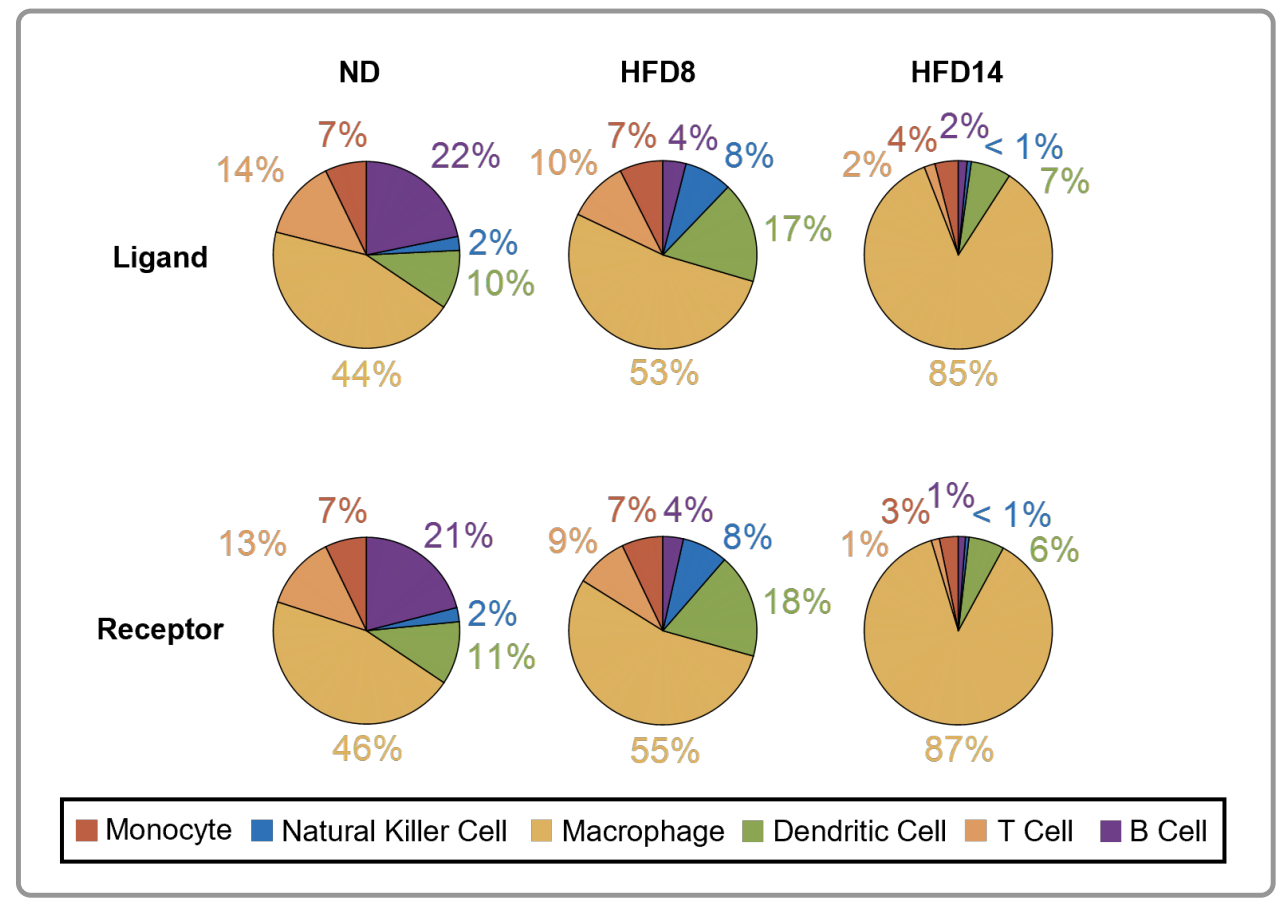

Supplementary Figure 4. Cell type contributions to ligand and receptor activity. 